\title{
A DETAILED ANALYTICAL STUDY OF NON-LINEAR SEMICONDUCTOR DEVICE MODELLING
}

\author{
UMESH KUMAR \\ Department of Electrical Engineering, I.I.T., Havz KHAS, New Delhi-110016, India \\ (Received March 6, 1995; in final form April 3, 1995)
}

This paper presents a detailed analytical study of Gunn, SCR, and p-n junction and of the physical processes that occur inside. Based on the properties of these devices, models for Gunn, SCR, and p-n junction diode have been developed. The results of computer simulated examples have been presented in each case. The non-linear lumped model for Gunn is a unified model as it describes the diffusion effects as the -domain traves from cathode to anode. An additional feature of this model is that it describes the domain extinction and nucleation phenomena in Gunn dioder with the help of a simple timing circuit. The non-linear lumped model for SCR is general and is valid under any mode of operation in any circuit environment. The memristive circuit model for $\mathrm{p}-\mathrm{n}$ junction diodes is capable of simulating realistically the diode's dynamic behavior under reverse, forward and sinusiodal operating modes. The model uses memristor, the charge-controlled resistor to mimic various second-order effects due to conductivity modulation. It is found that both storage time and fall time of the diode can be accurately predicted.

\section{INTRODUCTION}

Non linearity is an inherent property of semiconductor devices. Modern devices like the Gunn, SCR, IMA, and IMPATT devices, etc., are strongly non-linear. These devices exhibit complex dynamic behaviors. In order to design a circuit involving these devices, it does not suffice just to know the terminal behavior of the device. It becomes all the more necessary to design the device or model it properly. Though no general theory on device modelling is available, we can establish a set of systematic approximations to proceed from a description of the general subregion to a lumped model for the subregion. In the present analysis, first a general subregion is selected. Next, it is determined what physical processes and morphologic characteristics dominate the behavior of the subregion. Next, the form of equations is postulated that define the dominant factors on distributed basis, that is, with independent space and time variables. The postulated equations are combined. Approximations are then made, which in essence permit the removal of dependence on continuous space variables. The result is that the device is now approximated by a set of differential equations with independent variable time. The techniques available for network analysis and synthesis can be used to generate a symbolic model that will be network-like in form and therefore amenable to all the analysis and physical reasoning used with networks. 


\section{SEMI CONDUCTOR DEVICE MODELLING VIA BASIC NONLINEAR CIRCUIT ELEMENTS}

In addition to understanding of terminal behavior of a device, it also comes important to understand the interrelationship among the physical processes, device geometry, and terminal behavior. By developing a model to describe physical processes occuring in a device and its terminal behavior, an attempt is made to mimick the actual behavior of the device. Simplifying assumptions make a compromise between accuracy and complexity of the model.

\section{The Art of Device Modelling}

Circuit models of devices are obtained via two basic approaches-the physical approach and the black box approach.

\section{(A) Physical Approach}

The most reliable and logical approach is a careful study of the physics and operating mechanisms of the device. The following steps are involved in the physical approach to device modelling.

(i) Device physics analysis and partitioning

It consists of identifying the important physical variables, phenomena, and operating mechanism through a careful analysis of the device physics. This information can be used to partition the internal structure of the device into as many geometrically distinct regions as possible, such that each unit can be modelled separately.

(ii) Physical equation formulation

It consists of formulating the relevant physical equations that relate the internal physical variables in the partitioned units to each other and to the external terminal currents and voltages.

(iii) Equation simplification and solution

It consists of solving the equations formulated in step (ii). Since these equations are generally non-linear partial differential equations, no explicit closed form solution is generally possible. Lumped approximations are obtained by reducing the partial differential equations to ordinary differential equations.

(iv) Non-linear network synthesis

If step (iii) cannot be reduced to a lumped model, then step (iv) consists of synthesising a circuit model that uses both lumped and distributed (transmission lines) circuit elements. 


\section{(B) Black Box Approach}

If device physics and operating mechanisms are not well understood or when the device is so complex that a physical approach is not possible, we resort to this approach. The following basic steps are involved in the Black Box Approach.

(i) Experimental observation

(ii) Mathematical modelling

(iii) Model validation

(iv) Non-linear network synthesis.

The present study is restricted to physical approach only.

\section{CONCEPT OF A DEVICE MODEL-ITS CHARACTERISTICS}

Let $\mathrm{D}$ describe a device. It is physically impossible to measure the generally infinite collection $F(D)$ of admissible voltage - current signal pairs. Our approach is to develop a device model M(D) made of some well defined set of ideal circuit elements so that each admissible voltage current pair associated with $M(D)$ represents a good approximation to a corresponding $(v(t), i(t))$ measured from $D$.

A circuit model of $D$ is hence not an equivalent circuit of $D$ because no physical device can be exactly mimicked by a circuit or mathematical model.

In fact, depending on the application (e.g., amplitude and frequency of operation) a given device may have many distinct models. The best model in the given situation is the simplest model capable of yielding realistic solutions.

\section{Two Terminal Elements and Their Constitutive Relations}

Although it is impossible to measure and store all admissible voltage-current signal pairs $f(d)$ associated with a device $D$, we can define a hypothetical or ideal circuit element $\in$ whose associated collection $f(\epsilon)$ of admissible voltage-current pairs is generated by a prescribed algorithm hereforth called the constitutive relation of $\epsilon$.

The constitutive relationship of a two-terminal element $\in$ may be expressed in any convenient compact form. The constitutive relation of the three standard linear elements $(R, L$ and $C)$ can be expressed by

$$
\begin{aligned}
& \mathrm{v}=\mathrm{iR} \quad \text { or } \mathrm{i}=\mathrm{Gv} \\
& v=L \frac{d i}{d t} \quad \text { or } \quad i(t)=\frac{1}{L} \int_{-\infty}^{\tau} v(\tau) d \tau \\
& =\frac{\psi(\mathrm{t})}{\mathrm{L}} \\
& \mathrm{i}=\mathrm{C} \frac{\mathrm{dv}}{\mathrm{dt}} \quad \text { or } \quad \mathrm{v}(\mathrm{t})=\frac{1}{\mathrm{C}} \int_{-\infty}^{\tau} \mathrm{i}(\tau) \mathrm{d} \tau=\frac{1}{\mathrm{C}} \mathrm{q}(\mathrm{t})
\end{aligned}
$$

A non-linear current-controlled resistor can be described by $\mathrm{v}=\mathrm{v}(\mathrm{i})$ 
A non-linear voltage-controlled resistor can be described by $\mathrm{i}=\mathrm{i}(\mathrm{v})$ or implicitly by $f_{R}(v, i)=0$

The nonlinear inductor and capacitor can be described by

$f_{\mathrm{L}}(\psi, \mathrm{i})=0$ and $f_{\mathrm{c}}(\mathrm{q}, \mathrm{v})=0$

For more complex elements, the constitutive relation will generally consist of a system of non-linear, algebraic, differential, (ordinary or partial) and/or integral equations involving not only the variable $v$ and $i$, their higher order derivatives, and higher order integrals but also internal variable, their derivatives, and integrals.

\section{Lumped Versus Distributed Element}

A two-terminal element $\in$ is said to be lumped if its constitutive relation can be expressed by a finite number of equations involving only algebraic, ordinary differential and integral operation on the instantaneous values of the terminal variables $(v, i)$ and/or a finite number of additional internal variables $\left(X_{1}\right.$, $\left.\mathrm{X}_{2} \ldots \ldots \mathrm{X}_{\mathrm{n}}\right)$. Otherwise $\in$ is said to be distributed.

The constitutive relation of any lumped 2-terminal element consists of equations of the form,

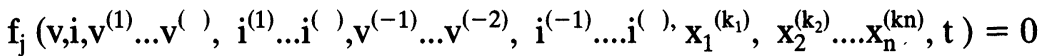

$$
\begin{aligned}
& (\mathrm{j}=1,2 \ldots . ., \mathrm{m})
\end{aligned}
$$

A lumped 2-terminal element $\in$ is said to be linear if $f_{1}(\cdot), f_{2}(\cdot) \ldots . f_{m}(\cdot)$ are linear functions of all variables at any time t. Otherwise $\in$ is said to be non-linear.

In non-linear network synthesis the specification is the prescribed constitutive relation and the solution is a network having 2-external terminals made of a prescribed set of building blocks.

\section{Four Basic Elements}

A two-terminal element $\in$ is called a 2-terminal Resistor, Inductor, Capacitor, Memristor if its constitutive relation can be expressed by an algebraic relationship denoted symbolically by

$$
\begin{array}{lll}
\mathrm{f}_{\mathrm{R}}(\mathrm{u}, \mathrm{i}, \mathrm{t})=0 & ; & \mathrm{v}-\mathrm{i} ; \mathrm{R} \\
\mathrm{f}_{\mathrm{l}}(\psi, \mathrm{i}, \mathrm{t})=0 & ; & \psi-\mathrm{i} ; \mathrm{L} \\
\mathrm{f}_{\mathrm{c}}(\mathrm{q}, \mathrm{v}, \mathrm{t})=0 & ; & \mathrm{q}-\mathrm{v} ; \mathrm{C} \\
\mathrm{f}_{\mathrm{m}}(\mathrm{q}, \psi, \mathrm{t})=0 & ; & \mathrm{q}-\psi ; \mathrm{M}
\end{array}
$$

\section{Memristor-The Mixing Element}

A IV element called memristor has been postulated to fill the missing link in the 4-element diagram. A charge controlled memristor can also be described by $\mathrm{v}=$ $R(q)$ i 
where

$R(q)=\frac{d \psi(q)}{d t}$ is called the memristor.

\section{NON-LINEAR LUMPED CIRCUIT MODEL FOR GUNN}

\section{Introduction to Gunn and its various modes}

In a voltage-controlled DNR like Gunn, the decrease in current density with an increase in electric field is brought about by decreasing drift velocity of electrons by increasing the field.

The current density is proportional to the density of carriers as well as their drift velocity $\left(J=n \sigma v_{d}\right)$. The mechanism that causes a fall in drift felocity of electrons with the rise in electric field across the sample as it crosses over a limit of some thousand volts/cm is a well known RWH mechanism. In a Gunn, when field exceeds certain threshold value, DNR is observed.

The product $\mathrm{n}_{\mathrm{o}} \mathrm{L}$ gives the condition for formation of stable domains. The criterion for different modes of operation is

(i) if $\mathrm{n}_{\mathrm{o}} \mathrm{L}<10^{12}$, table domains are not formed, though space charge accumulations are formed. This is called space charge accumulation mode.

(ii) $\mathrm{n}_{\mathrm{o}} \mathrm{L}>10^{12}$, if a resonant circuit is connected across the Gunn, various other modes of operation are possible.

\section{(a) Transit Time Mode}

When the $\mathrm{fL}$ product is approximately equal to the average carrier drift velocity ( $\mathrm{fL}$ is $10^{7} \mathrm{~cm} / \mathrm{sec}$ ) the sample operates in this mode. In this mode, the high field domain is nucleated at the cathode and travels the full lengths of samples to the anode.

Frequency of oscillation is given by

$f=\frac{U_{\text {dom }}}{L^{*}} \quad L^{*}-$ effective length of sample

(b) Quenched Domain Mode ( $\mathrm{fL}>2 \times 10^{7} \mathrm{~cm} / \mathrm{sec}$ )

A bulk $G_{a}-A_{s}$ oscillator can oscillate at frequencies higher than the transit time frequency if the high field domain is quenched before it reaches the anode. Dipole domain quenching occurs when the bias voltage across the samples is reduced below $V_{s}$. The frequency of oscillation is frequency of the resonant circuit.

(c) Inhibited Mode

If the total voltage across an RWH device is below threshold at the instant a dipole disappears at the anode, the formation of a new dipole is delayed until the voltage 
rises above threshold. If the new domain is inhibited from starting for a time equal to the domain transit time, then the waveform will be approximately a square wave. The frequency of resonant circuit is tuned to half the transit time frequency.

(d) LSA Mode

In a uniformly doped semiconductor without any internal space charges, the internal field would be uniform and simply proportional to the applied voltage. The current would be proportional to the drift velocity at this field level. Hence, current-voltage characteristics of the device would be the same in shape as the velocity field curve.

If this is coupled to an external resonant circuit, this could excite oscillations at the frequency of a resonant circuit. In this mode, the electric field across the diode rises from below threshold and falls back again so quickly that space charge distribution associated with high-field domain does not have enough time to form. Only primary layers form near the cathode, while the rest of the samples remains fairly homogeneous.

\section{Assumptions made in the Non-linear Model developed for Gunn}

This model is a verfied circuit model. It however, makes the following assumptions

(i) The parameters associated with the model are fixed for each device, regardless of the mode of operation or external circuits.

(ii) There exists a high field domain with a single local maximum that propagates without change of shape with a domain velocity $V_{D}(t)$ from cathode to anode.

(iii) Quasi-Static assumption

We introduce a new variable $y$ as the distance measured in a system of coordinates moving in the direction of the electron flow with the domain velocity $\mathrm{V}_{\mathrm{D}}(\mathrm{t})$

$$
\begin{aligned}
& \mathrm{y}=\mathrm{x}-\int_{o}^{t} \mathrm{~V}_{o}(t) d t \\
& \mathrm{t}^{\prime}=\mathrm{t}, \quad \frac{\sigma \mathrm{E}}{\sigma \mathrm{T}^{\prime}}=0
\end{aligned}
$$

\section{Lumped Circuit Model for Domain Dynamics}

Let us take a 1-D structure of length $\mathrm{L}$, area $\mathrm{A}$, dielectric constant $\in$, and a uniform donor concentration $n_{o}$ (Fig. 1(a)). $n_{o} L>10^{12}$ enables support of high-field domains consisting of an accumulation layer with carrier concentration $n>n_{o}$ and a depletion layer with carrier concentration $n<n_{o}$ (Fig. 1(b)). The corresponding dipole-induced field distribution is shown in (Fig. 1(c)). These figures give shapes of $\mathrm{n}(\mathrm{x})$ and $\mathrm{F}(\mathrm{x})$ at one instant of time. As the domain grows in size, it propagates from the cathode $(x=O)$ to the anode $(x=L)$ with an instantaneous velocity $v_{D}(t)$. 


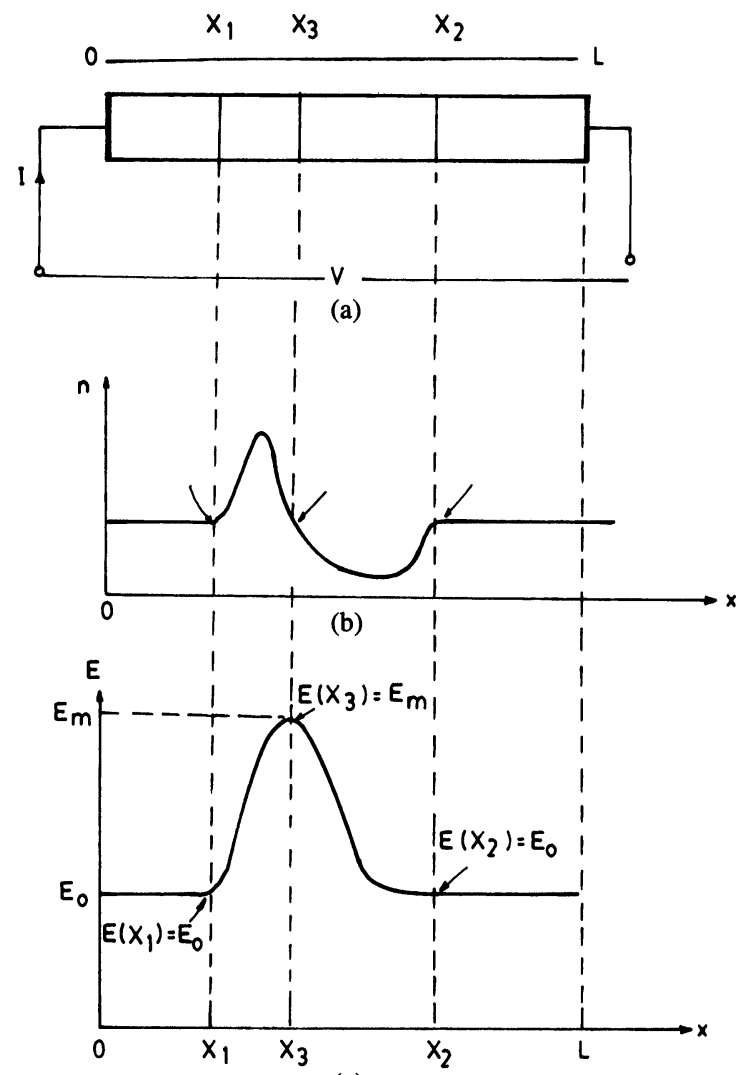

(c)

FIGURE 1 (a): A one-dimensional Gunn Diode (b): Carrier concentration at one instant of time (c); Electric field distribution inside Gunn Diode at one instant of time.

Shapes of $n(x)$ and $E(x)$ at different instant of time are governed by complex dynamics and affect device current and voltage as we shall see

$\mathrm{n}(\mathrm{x})=\mathrm{n}_{\mathrm{a}}(\mathrm{x}) \quad ; \quad \mathrm{x}_{1}<\mathrm{x}<\mathrm{x}_{3}$

$\mathrm{n}(\mathrm{x})=\mathrm{n}_{\mathrm{d}}(\mathrm{x}) \quad ; \mathrm{X}_{3}<\mathrm{X}<\mathrm{X}_{2}$

One can write,

$$
\mathrm{n}_{\mathrm{a}}=\mathrm{n}_{\mathrm{a}}(\mathrm{E}) \quad\left(\mathrm{E}_{\mathrm{o}}, \mathrm{E}_{\mathrm{m}}\right)
$$

and $\mathrm{n}_{\mathrm{d}}=\mathrm{n}_{\mathrm{d}}(\mathrm{E}) \quad\left(\mathrm{E}_{\mathrm{o}}, \mathrm{E}_{\mathrm{m}}\right)$

as single valued function of $\mathrm{E}$.

Description of the Model-Evaluation of Associated Model Parameters and Functions

1. Cathode to anode capacitance; $C_{1}=\frac{\in A}{L}$ 
2. Domain capacitance; $\mathrm{C}_{2}=\frac{\in \mathrm{A}}{\mathrm{W}}$

$\mathrm{W}=$ Average domain width $=\mathrm{Lt} \int_{0}^{\mathrm{T}}\left|\mathrm{x}_{2}(\mathrm{t})-\mathrm{x}_{1}(\mathrm{t})\right| \mathrm{dt} \quad \mathrm{T} \rightarrow \infty$

3. Nonlinear resistor, $\mathbf{R}$

$$
I_{R}=G\left(V_{R}\right)=A_{q} n_{o} v\left(V_{R} / L\right)
$$

where $\mathrm{V}(\mathrm{E})=\mathrm{V}\left(\mathrm{V}_{\mathrm{R}} / \mathrm{L}\right) ; \mathrm{E}=\mathrm{V}_{\mathrm{R}} / \mathrm{L}$

4. Non-linear current controlled source

Terminal current $I_{D}$ of the controlled current source depends on the instantaneous value of capacitor voltage $V_{1}(t)$ and $V_{2}(t)$ along with the device terminal current $I(t)$ in accordance with the following non-linear relationship -

$\mathrm{I}_{\mathrm{D}}=\mathrm{I}_{\mathrm{D}}\left(\mathrm{V}_{1}, \mathrm{~V}_{2}, \mathrm{I}\right)=\mathrm{C}_{2} \mathrm{~F}\left(\mathrm{~V}_{1}, \mathrm{~V}_{2}\right)-\mathrm{I}$

$F\left(V_{1}, V_{2}\right)=n_{o}\left\{\int_{V_{1 / L}}^{E m}\left|\frac{v\left(V_{1} / L\right)-V(E)}{n_{a}(E)-n_{o}}\right| d E+\int_{V_{1 / L}}^{E m} \frac{v\left(V_{1} / L\right)-V(E)}{n_{o}-n_{d}(E)} d E\right\}$

$\mathrm{D}(\mathrm{E}) \rightarrow$ Non-linear function of $\mathrm{E}$.

$n_{a}(E), n_{d}(E), E_{m}, D(E)$ are found for each value of $\left(v_{1}, v_{2}\right)$ as:

$\mathrm{G}\left(\mathrm{E}_{m} ; \mathrm{V}_{1}, \mathrm{~V}_{2}\right)=0$

$G\left(E_{m} ; V_{1}, V_{2}\right)=V_{2}-\frac{E}{q}\left\{\int_{V_{1 / L}}^{E m} \frac{E-V_{1} / L}{n_{a}(E)-n_{o}} d E+\int_{V_{1} / L}^{E m} \frac{E-V_{1} / L}{n_{o}-n_{d}(E)} d E\right\}$

Accumulation layer carrier concentration $n_{a}(E)$

For each given value of $\left(V_{1}, V_{2}\right) n_{a}(E)$ is obtained by solving the following scalar non-linear ordinary differential equation

$\frac{\mathrm{dn}_{\mathrm{a}}}{\mathrm{dE}}=\mathrm{N}_{\mathrm{a}}\left(\mathrm{n}_{\mathrm{a}}, \mathrm{E} ; \mathrm{V}_{19} \mathrm{~V}_{\mathrm{D}}\right)$

where, $N_{a}\left(n_{a}, E ; V_{1}, V_{D}\right) \triangleq n_{a}\left[V(E)-V_{D}\right]+n_{o}$

$\left[\underline{V_{D}-V\left(V_{1} / L\right)}\right]-\frac{q}{E}\left(n_{a}-n_{o}\right) n_{a} D^{\prime}(E) / q / E\left(n_{a}-n_{o}\right) D(E)$

$n_{a}(E) \mid E=E_{o}=v_{1} / L=n_{o}$ 
$\mathrm{n}_{\mathrm{a}}(\mathrm{E}) \mid \mathrm{E}=\mathrm{E}_{\mathrm{m}}=\mathrm{n}_{\mathrm{o}}$

$\mathrm{D}^{\prime}(\mathrm{E})$ is known

$\mathrm{V}(\mathrm{D})=\mathrm{V}_{\mathrm{D}}\left(\mathrm{V}_{1}, \mathrm{~V}_{2}\right)$ is domain velocity.

Since $8(a)$ is a non-linear scalar first-order differential equation, $8(\mathrm{c})$ and $8(\mathrm{~d})$ cannot be satisfied simultaneously for arbitrary values of $V_{D}$. Consequently, to solve for $\mathrm{n}_{\mathrm{a}}=\mathrm{n}_{\mathrm{a}}(\mathrm{E})$ we must choose an optimum value for $\mathrm{V}_{\mathrm{D}}$ in order that $8(\mathrm{c})$ and $8(d)$ are satisfied. Hence, we must solve a two-point boundary problem giving both $V_{D}$ and $n_{a}(E)$ as its solution.

To solve this two-point boundary value problem, we would use a 'shooting method',

$$
\mathrm{n}_{\mathrm{a}}(\mathrm{E})=\mathrm{n}_{\mathrm{a}}\left(\mathrm{E}, \mathrm{V}_{1}, \mathrm{E}_{\mathrm{m}}\right)=\mathrm{n}_{\mathrm{a}}\left(\mathrm{V}_{1}, \mathrm{~V}_{2}\right) \text { for } \mathrm{E}_{\mathrm{m}}=\mathrm{E}_{\mathrm{m}}\left(\mathrm{V}_{1}, \mathrm{~V}_{2}\right)
$$

Depletion Layer conc. $\mathrm{n}_{\mathrm{d}}(\mathrm{E})$ :

For each given value of $\left(V_{1}, V_{2}\right), n_{d}(E) V_{s}$ obtained by solving following the 'initial value problem'

$\frac{d n_{d}}{d E}=N_{d}\left(n_{d}, E ; V_{1}, V_{D}\right)$

$\left.\mathrm{N}_{\mathrm{d}}\left(\mathrm{n}_{\mathrm{d}}, \mathrm{E} ; \mathrm{V}_{1}, \mathrm{~V}_{\mathrm{D}}\right) \triangleq \mathrm{n}_{\mathrm{d}}\left[\mathrm{V}(\mathrm{E})-\mathrm{V}_{\mathrm{D}}\right]-\mathrm{n}_{\mathrm{o}}\left[\mathrm{V}_{\mathrm{D}}-\mathrm{V}_{1} / \mathrm{L}\right)\right]-$

$\frac{(q / E)\left(n_{d}-n_{o}\right) n_{d} D^{\prime}(E)}{(q / E)\left(n_{d}-n_{o}\right) D(E)}$

$\left.\mathrm{n}_{\mathrm{d}}(\mathrm{E})\right|_{\mathrm{E}}=\mathrm{E}_{\mathrm{o}}=\mathrm{V}_{\mathrm{1}} / \mathrm{L}=\mathrm{n}_{\mathrm{o}}$

$\mathrm{V}_{\mathrm{D}}=\mathrm{V}_{\mathrm{D}}\left(\mathrm{V}_{1}, \mathrm{~V}_{2}\right)$

$V_{D}$ is already obtained from equation $8(a)$ for given $\left(V_{1}, V_{2}\right) \cdot n_{d}$ depends on both $\mathrm{V}_{1}$ and $\mathrm{E}$ so,

$$
\mathrm{n}_{\mathrm{d}}(\mathrm{E})=\mathrm{n}_{\mathrm{d}}\left(\mathrm{E} ; \mathrm{V}_{1} ; \mathrm{E}_{\mathrm{m}}\right)
$$

Equations (7), (8) and (9) are coupled together; hence, we must solve them by iterative methods (as simultaneous equations)

Equation (7) is only a scalar algebraic equation and can be solved by NewtonRaphson or the Secant method. Once $n_{a}(E), n_{d}(E)$ and $E_{m}$ are found, $F\left(V_{1}, V_{2}\right)$ can be found.

Derivation of the Model

Let us call region $\mathrm{O}<\mathrm{x}_{1} \leq \mathrm{x} \leq \mathrm{x}_{2}<1$ as a domain region that contains a high-field domain at any instant $t$. The remaining region where $n=n_{o}$ and $E=E_{o}$ is referred to as the 'outside region'. Current density $J_{2}$ (inside the domain) $=J_{1}$ (outside the region) 
$q n v(E)+E \frac{\partial E}{\partial t}-q \frac{\partial}{\partial x} D(E) n=q n_{o} V\left(E_{o}\right)+E \frac{\partial E_{o}}{\partial t}$

$\frac{\partial\left(E-E_{o}\right)}{\partial t}=\frac{q n_{o}}{E}\left[V\left(E_{o}\right)-V(E)\right]+\frac{q}{E} \frac{\partial D(E) n}{\partial x}-V(E)\left[\frac{q}{E}\left(n-n_{o}\right)\right]$

Poisson equation:

$\frac{\partial E}{\partial X}=\frac{q}{E}\left(n-n_{o}\right) V_{2} \triangleq \int_{X_{1}}^{X_{2}}\left(E-E_{o}\right) d X$

$\mathrm{E}_{\mathrm{o}}=$ field outside domain

$\mathrm{E}$ = field inside domain

Integrating both sides of (11) from $\mathrm{X}_{1}$ to $\mathrm{X}_{2}$

$\frac{d V_{2}}{d t}=\int_{x_{1}}^{x_{2}} \frac{q n_{o}}{E}\left[V\left(E_{o}\right)-V(E)\right] d X+\underset{D\left(E\left(X_{1}\right)\right) n\left(X_{1}\right)}{\frac{q}{E}} \int_{E\left(X_{1}^{V}\right)}^{d}(D(E) n)-\int_{\left.X_{2}\right) n\left(X_{2}\right)}^{E} V(E) d E$

$\mathrm{E}\left(X_{1}\right)=\mathrm{E}\left(X_{2}\right)=E_{o}, n\left(X_{1}\right)=n\left(X_{2}\right)=n_{o} \frac{d v_{2}}{d t}=\int_{x_{2}}^{x_{2}} \frac{q n_{o}}{E}\left[V\left(E_{o}\right)-V(E)\right] d X$

Let $E$ has a single max at $X=X_{3}$ of $E=E_{m}$ value

$\frac{d V_{2}}{d t}=\int_{x_{1}}^{x_{3}} \frac{q n_{o}}{E}\left[V\left(E_{o}\right)-V(E)\right] d x+\int_{x_{3}}^{x_{2}} \frac{q n_{o}}{E}\left[V\left(E_{o}\right)-V(E)\right] D X$

using Poisson's equation:-

$\frac{d V_{2}}{d t}=\int_{E_{o}}^{E_{m}} \frac{n_{o}\left[V\left(E_{o}\right)-V(E)\right]}{n_{a}-n_{o}} d E+\int_{E_{o}}^{E_{m}} \frac{n_{o}\left[V\left(E_{o}\right)-V(E)\right]}{n_{o}-n_{d}} d E$

Using Poisson's equation in (12) (splitting integral $\mathrm{X}_{1}-\mathrm{X}_{2}$ into two parts; $\mathrm{X}_{1}-\mathrm{X}_{3}$ \& $\mathrm{X}_{3}-\mathrm{X}_{2}$ )

$V_{2}=\frac{E}{q} \int_{E_{o}}^{E_{m}} \frac{E-E_{o}}{\left(n_{a}-n_{o}\right)} d E+\frac{E}{q} \int_{E_{o}}^{E_{m}} \frac{E-E_{o}}{\left(n_{o}-n_{d}\right)} d E$

Let us derive an expression relating the external device current $I$ to the internal field. Total current outside the domain,

$I=A q n_{o} v\left(E_{o}\right)+E A \frac{d E_{o}}{d t}$ 
Corresponding voltage across the device is given by

$V=\int_{0}^{L} E(X) d X=\int_{0}^{L} E_{o} d x+\int_{0}^{L}\left(E-E_{o}\right) d x V=V_{1}+V_{2} ; V_{1}=E_{o} L$

These equations 15,16 , and 17 completely describe the dynamics of high-field domain in terms of external device current $I$ and $V$.

Applying KCL at upper node,

$I=A q n_{o} v\left(v_{1} / L\right)+\frac{E A}{L} \frac{d v_{1}}{d t}$

At lower node,

$\mathrm{I}+\mathrm{I}_{\mathrm{D}}=\mathrm{C}_{2} \frac{\mathrm{dv}_{2}}{\mathrm{dt}}$

$I+\left[C_{2} F\left(v_{1}, v_{2}\right)-I\right]=C_{2} \frac{d v_{2}}{d t}$

$\Rightarrow \frac{\mathrm{dv}_{2}}{\mathrm{dt}}=\mathrm{F}\left(\mathrm{v}_{1}, \mathrm{v}_{2}\right)$.

$\Rightarrow F\left(v_{1}, v_{2}\right)=n_{o}\left\{\int_{V_{1} / L}^{E_{m}} \frac{v\left(v_{1} / L\right)-V(E)}{n_{a}(E)-n_{o}} d E+\int_{V_{1} / L}^{E_{m}} \frac{v\left(V_{1} / L\right)-V(E)}{n_{o}-n_{d}(E)} d E\right\}$

Substituting $V_{1} / L$ for $E_{o}$ we get

$I=a q n_{o} v\left(v_{1} / L\right)+\frac{E A}{L} \frac{d v_{1}}{d t}$

Hence, our model is consistent with device physics. Also,

$V_{2}-\frac{E}{q}\left\{\int_{V_{1} / L}^{E_{m}} \frac{E-E_{o}}{\left(n_{a}-n_{o}\right)} d E+\int_{V_{1} / L}^{E_{m}} \frac{E-E_{o}}{n_{o}-n_{d}} d E=0\right\}$

$=\mathrm{G}\left(\mathrm{E}_{\mathrm{m}}, \mathrm{V}_{1}, \mathrm{v}_{2}\right)$ or $\mathrm{G}\left(\mathrm{E}_{\mathrm{m}}, \mathrm{V}_{1}, \mathrm{~V}_{2}\right)=0$

Finally $n_{a}, n_{d}$ satisfy following condition,

$\mathrm{n}\left(\mathrm{X}_{1}\right)=\mathrm{n}_{\mathrm{a}}\left(\mathrm{E}_{\mathrm{o}}\right)=\mathrm{n}_{\mathrm{o}}$

$\mathrm{n}\left(\mathrm{X}_{3}\right)=\mathrm{n}_{\mathrm{a}}\left(\mathrm{E}_{\mathrm{m}}\right)=\mathrm{n}_{\mathrm{o}}$

$\mathrm{n}\left(\mathrm{X}_{2}\right)=\mathrm{n}_{\mathrm{d}}\left(\mathrm{E}_{\mathrm{o}}\right)=\mathrm{n}_{\mathrm{o}}$ 
Since the high field domain grows as it propagates from the cathode $(\mathrm{X}=\mathrm{O})$ to anode $(X=L)$ with an instantaneous velocity $V_{D}(t)$, the electric field $E$ and the electron density $n$ depend upon both 'time' $t$ and 'space' variable $x$. The exact form of $n_{a}, n_{d}$, and $E$ must be obtained by solving the partial differential equation. Here we would make some simplifying assumptions. For the general field-dependent diffn. case, the external device current I can be expressed as,

$\frac{I}{A q}=n v(E)-\frac{\partial(D n)}{\partial X}+\frac{E}{q} \frac{\partial E}{\partial t}$

Let us introduce, $y \triangleq X-\int_{o}^{t} V_{D}(t) d t, t^{\prime}=t$

This was our initial assumption (ii)

$$
\begin{aligned}
& \frac{\partial(D n)}{\partial y}=n V(E)-\frac{I}{A q}+\frac{E}{q}\left[-V_{D} \frac{\partial E}{\partial Y}+\frac{\partial E}{\partial t^{\prime}}\right] I=\partial q n_{o} v\left(V_{1} / L\right), \frac{\partial E}{\partial t^{\prime}}=0 \\
& \frac{\partial(D n)}{\partial y}=n v(E)-n_{o} v\left(V_{1} / L\right)-\frac{E}{q} V_{D} \frac{\partial E}{\partial y}
\end{aligned}
$$

Poisson's equation $\frac{\partial E}{\partial y}=\frac{\partial E}{\partial X}, \frac{\partial X}{\partial y}=\frac{q}{E}\left(n-n_{o}\right)$

$\frac{\partial(D n)}{\partial y}=\frac{\partial(D n)}{\partial E}, \frac{\partial E}{\partial y}=\frac{q}{E}\left(n-n_{o}\right)\left|D \frac{\partial n}{\partial E}+n \frac{\partial D}{\partial E}\right|$

Substituting 18, 19, and 20 in

$$
\frac{\mathrm{dn}}{\mathrm{dE}}=\frac{\mathrm{n}\left[\mathrm{v}(\mathrm{E})-\mathrm{V}_{\mathrm{D}}\right]-\mathrm{n}_{\mathrm{o}}\left[\mathrm{V}_{\mathrm{D}}-\mathrm{V}\left(\mathrm{V}_{1} / \mathrm{L}\right)\right]-\mathrm{q} / \mathrm{E}\left(\mathrm{n}-\mathrm{n}_{\mathrm{o}}\right) \mathrm{n} \mathrm{D}^{\prime}(\mathrm{E})}{(\mathrm{q} / \mathrm{E})\left(\mathrm{n}-\mathrm{n}_{\mathrm{o}}\right) \mathrm{D}(\mathrm{E})}
$$

This completes our derivation of model.

\section{Derivation of Simplified Models}

Let us recast (20a) into

$$
\left(1-\frac{n_{o}}{n} \frac{d\left(\frac{n}{n_{o}}\right)}{d E}=\frac{E}{q n_{o}}\left[\frac{V(E)-V_{D}}{D(E)}\right]+\frac{E}{q}\left[\frac{V_{D}-V\left(V_{1} / L\right)}{n D(E)}\right]-\frac{\left(\frac{n}{-n_{o}}-1\right)}{D(E)} D^{\prime}(E)\right.
$$

Integrating both sides from $E_{o}$ to $E$ we get,

$$
\frac{n}{n_{o}}-\ln \frac{n^{-1}}{n_{o}}=\frac{E}{q n_{o}} \int_{E_{o}}^{E_{m}} \frac{V(E)-V_{D}}{D(E)} d E+\frac{E}{q}\left[V_{D}-V\left(V_{1} / L\right)\right]
$$




$$
\begin{aligned}
& \int_{E_{o}}^{E_{m}} \frac{1}{n D(E)} d E-\int_{E_{o}}^{E} \frac{D^{\prime}(E)}{D(E)}\left(\frac{n}{n_{o}}-1\right) d E \\
& \text { At } E=E_{m}, n=n_{o} \\
& 0=\frac{E}{q n_{o}} \int_{E_{o}}^{E_{m}} \frac{V(E)-V_{D}}{D(E)} d E+\frac{E}{q}\left[V_{D}-V\left(V_{1} / L\right)\right] \int_{E_{o}}^{E_{m}} \frac{1}{n D(E)} d E \int_{E_{o}}^{E_{m}} \frac{D^{\prime}(E)}{D(E)}\left(\frac{n}{n_{o}}-1\right) d E
\end{aligned}
$$

Accumulation layer:

$0=\frac{E}{q n_{o}} \int_{E_{o}}^{E_{m}} \frac{\left[V(E)-V_{D}\right]}{D(E)} d E+\frac{\epsilon}{q}\left[V_{D}-V\left(V_{1} / L\right)\right] \int_{E_{o}}^{E_{m}} \frac{1}{n_{a} D(E)} d E-\int_{E_{o}}^{E_{m}} \frac{D^{\prime}(E)}{D(E)} \frac{\left(n_{a-1}\right)}{n} d E$

Depletion layer:

$0=\frac{\epsilon}{q n_{o}} \int^{E_{m}} \frac{V(E)-V_{D}}{E_{o} D(E)} d E+\frac{\epsilon}{q}\left[V_{D}-V\left(V_{1} / L\right)\right] \int_{E_{o}}^{E_{m}} \frac{1}{n_{d} D(E)} d E-\int_{E_{o}}^{E_{m}} \frac{D(E)}{D(E)}\left(\frac{n_{d}}{n_{o}}-1\right) d E$

Subtracting,

$\left.v_{D}=V\left(V_{1} / L\right)-\frac{q}{\epsilon}\left\{\frac{\int_{V_{1} / L}^{E_{m}} \frac{D^{\prime}(E)}{D(E)}\left[n_{a}(E)-n_{d}\right] d E}{\int_{V_{1} / L}^{E_{m}} \frac{1}{D(E)}\left[\frac{1}{n_{d}(E)}-\frac{1}{n_{a}(E)}\right] d E}\right\}=V_{D}(V)_{1}, V_{2}\right)$

Special case:

1. Constt. Diffusion $\quad \mathrm{D}(\mathrm{E})=\mathrm{D}$

$D^{\prime}(E)=0 \Rightarrow V_{D}=V\left(V_{1} / L\right)$

Substracting for $V_{D}$

$$
\begin{aligned}
& \frac{n_{a}}{n_{o}}-\ln \frac{n_{a}}{n_{o}}-1=\frac{E}{q n_{o}} \int_{D_{V_{1} / L}}^{E}\left[V(E)-V\left(V_{1} / L\right)\right]_{d E}=h\left(E ; V_{1}\right) \\
& \frac{n_{d}}{n_{o}}-\ln \frac{n_{d}}{n_{o}}-1=\frac{E}{q n_{o} D} \int_{V_{1} / L}^{E}\left[V(E)-V\left(V_{1} / L\right)\right] d E=h\left(E ; V_{1}\right)
\end{aligned}
$$


Substracting for $n_{a}-n_{d}$ in . . . we can find $E_{m}$ by which gives $F\left(V_{1}, V_{2}\right)$ and thus $\mathrm{I}_{\mathrm{D}}$

2. Zero diffusion case $\mathrm{D}(\mathrm{E})=0$.

$\mathrm{n}_{\mathrm{a}} \Rightarrow \infty, \mathrm{n}_{\mathrm{d}} \Rightarrow 0 \quad \mathrm{~V}_{2}=\frac{\epsilon}{\mathrm{qn} \mathrm{n}_{\mathrm{o}}} \int_{\mathrm{V}_{1} / \mathrm{L}}^{\mathrm{E}_{\mathrm{m}}}\left[\mathrm{E}-\mathrm{V}_{1} / \mathrm{L}\right] \mathrm{dE}$

We can solve for $G\left(E_{m} ; V_{1}, V_{2}\right)=0$. This gives $E_{m}$ as

$$
\begin{aligned}
& \mathrm{E}_{\mathrm{m}}=\frac{\mathrm{V}_{1}}{\mathrm{~L}}+\underbrace{}_{\mathrm{E}_{\mathrm{m}}} \sqrt{\left[\left(\frac{2 \mathrm{qn}_{\mathrm{o}}}{\mathrm{E}}\right) \mathrm{V}_{2}\right]} \\
& F\left(V_{1}, V_{2}\right)=\int_{\mathrm{V}_{1} / \mathrm{L}}\left[\mathrm{V}\left(\mathrm{V}_{1} / \mathrm{L}\right)-\mathrm{V}(\mathrm{E})\right] \mathrm{dE} \\
& \mathrm{I}_{\mathrm{D}}=\mathrm{C}_{2} \mathrm{~F}\left(\mathrm{~V}_{1}, \mathrm{~V}_{2}\right)-\mathrm{I}=\mathrm{C}_{2} \int_{\mathrm{V}_{1} / \mathrm{L}}^{\mathrm{V}_{1} / \mathrm{L}+\frac{2 \mathrm{qn}^{\circ} \mathrm{V}_{2}}{-\mathrm{E}}}\left[\mathrm{V}\left(\mathrm{V}_{1} / \mathrm{L}\right)-\mathrm{V}(\mathrm{E})\right] \mathrm{dE}-\mathrm{I}
\end{aligned}
$$

Complete Lumped Circuit Model Including Domain Extinction (at the anode) and Nucleation

The Model developed so far is valid as long as the domain is extinguished by the external circuit constraints before it reaches the anode such as in the quenched domain mode or when a nature domain does not exist such as in LSA mode. In the transit time mode or delayed domain mode, the above is valid only during the time the domain has not reached the anode.

In order to model the domain extinction at the anode, we devised a timing cirucit that tracks the domain motion and causes the domain capacitor to discharge quickly, wherever the domain reaches the anode. This timing cirucit is added to the original Gunn Model.

\section{Timing Circuit}

It has one non-linear-controlled current source $\mathrm{I}_{\mathrm{C}}$ and three voltage-controlled resistors $R_{1}\left(V_{1}\right), R_{2}\left(V_{2}\right)$ and $R_{3}\left(V_{3}\right)$. These resistors behave like relays and should be described by a separate sub-routine using logic statements. Hence, considrable computer time can be saved.

$$
\mathrm{I}_{\mathrm{C}}=\operatorname{Aq} \mathrm{n}_{\mathrm{o}} \mathrm{V}_{\mathrm{D}}\left(\mathrm{V}_{1}, \mathrm{~V}_{2}\right)
$$

In constant diffusion case

$$
\begin{aligned}
& V_{D}=V\left(V_{1} / L\right) \\
& I_{C}=\operatorname{Aq~} n_{o} V\left(V_{1} / L\right)=I_{R}
\end{aligned}
$$


which is a linear controlled current source

$$
\begin{aligned}
& R_{1}\left(V_{1}\right)= \begin{cases}0 & V_{1}>E_{t} L=V_{1} t \\
\infty & V_{1}<E_{t} L=V_{1} t\end{cases} \\
& R_{2}\left(V_{2}\right)= \begin{cases}0 & V_{2}=0 \\
\infty & V_{2} \neq 0\end{cases} \\
& R_{3}\left(V_{3}\right)= \begin{cases}\tau_{d} / C_{2} V_{3}>A q n_{o} L \\
\infty & V_{3}<A q n_{o} L\end{cases} \\
& C_{2} \rightarrow \text { Domain capacitance } \\
& \tau_{d} \rightarrow \text { Average time constant for a domain to collapse. }
\end{aligned}
$$

\section{SCR Circuit Model}

The model contains 6 elements, namely, 3 non-linear capacitors and 3 non-linear voltage-controlled current sources. The incremental capacitances describing the capacitors and the functions describing the controlled sources are completely specified by the following equations:

Non-linear capacitors:

$$
\mathrm{C}_{\mathrm{j}}\left(\mathrm{V}_{\mathrm{j}}\right)=\mathrm{C}_{\mathrm{oj}}\left|-\mathrm{V}_{\mathrm{j}}\right|^{-1 / \mathrm{mj}}+\frac{\mathrm{I}_{\mathrm{sij} \tau_{\mathrm{j}}}}{\mathrm{V}_{\mathrm{T}}} \mathrm{e}^{\mathrm{V}_{\mathrm{j}} / \mathrm{V}_{\mathrm{T}}}
$$

Non-linear voltage controlled current sources:

$$
\begin{aligned}
& \left.\mathrm{f}_{\mathrm{A}}\left(\mathrm{V}_{1}, \mathrm{~V}_{2}\right)=\left|1\left(1+\gamma_{1}\right) \mathrm{I}_{\mathrm{S} 11}+\mathrm{I}_{\mathrm{S} 15}\right| \mathrm{e}^{\mathrm{V}_{1} / \mathrm{V}_{\mathrm{T}}}-1\right)+\mathrm{I}_{\mathrm{S} 21}\left(\mathrm{e}^{\mathrm{V}_{1} / 2 \mathrm{~V}_{\mathrm{T}}}-1\right)-\mathrm{I}_{\mathrm{S} 12}\left(\mathrm{e}^{\mathrm{V}_{2} / \mathrm{V}_{\mathrm{T}}}-1\right) \\
& \mathrm{f}_{\mathrm{B}}\left(\mathrm{V}_{1}, \mathrm{~V}_{2}, \mathrm{~V}_{3}\right)=\mathrm{I}_{\mathrm{S} 11}\left(\mathrm{e}^{\mathrm{V}_{1} / \mathrm{V}_{\mathrm{T}}}-1\right)-\mid\left(1+\gamma_{1}\right) \mathrm{I}_{\mathrm{S} 12}+\mathrm{I}_{\mathrm{S} 14}\left(1+\gamma_{2}\right) \\
& \left(\mathrm{e}^{\mathrm{V}_{2} / \mathrm{V}_{\mathrm{T}}}-1\right)-\mathrm{I}_{\mathrm{S} 22}\left(\mathrm{e}^{\mathrm{V}_{2} / 2 \mathrm{~V}_{\mathrm{T}}}-1\right)+\mathrm{I}_{\mathrm{S} 13}\left(\mathrm{e}^{\mathrm{V}_{3} / \mathrm{V}_{\mathrm{T}}}-1\right) \\
& \mathrm{f}_{\mathrm{C}}\left(\mathrm{V}_{2}, \mathrm{~V}_{3}\right)=-\mathrm{I}_{\mathrm{S} 14}\left(\mathrm{e}^{\mathrm{V}_{2} / \mathrm{V}_{\mathrm{T}}}-1\right)+\left|\left(1+\mathrm{V}_{2}\right) \mathrm{I}_{\mathrm{S} 13}+\mathrm{I}_{\mathrm{S} 16}\right|\left(\mathrm{e}^{\mathrm{V}_{3} / \mathrm{V}_{\mathrm{T}}}-1\right)+ \\
& \mathrm{I}_{\mathrm{S} 23}\left(\mathrm{e}^{\mathrm{V}_{3} / 2 \mathrm{~V}_{\mathrm{T}}}-1\right)
\end{aligned}
$$

These equations are completely specified by following 22 model parameters:

(a) Physical parameters:

Junction contact potential $\left(0-5<\mathrm{V}_{\mathrm{j}}<1.2 \mathrm{~V}\right) \mathrm{V}_{\mathrm{T}}=\frac{\mathrm{kT}}{\mathrm{q}}=26 \mathrm{mV}$.

(b) DC parameters: 


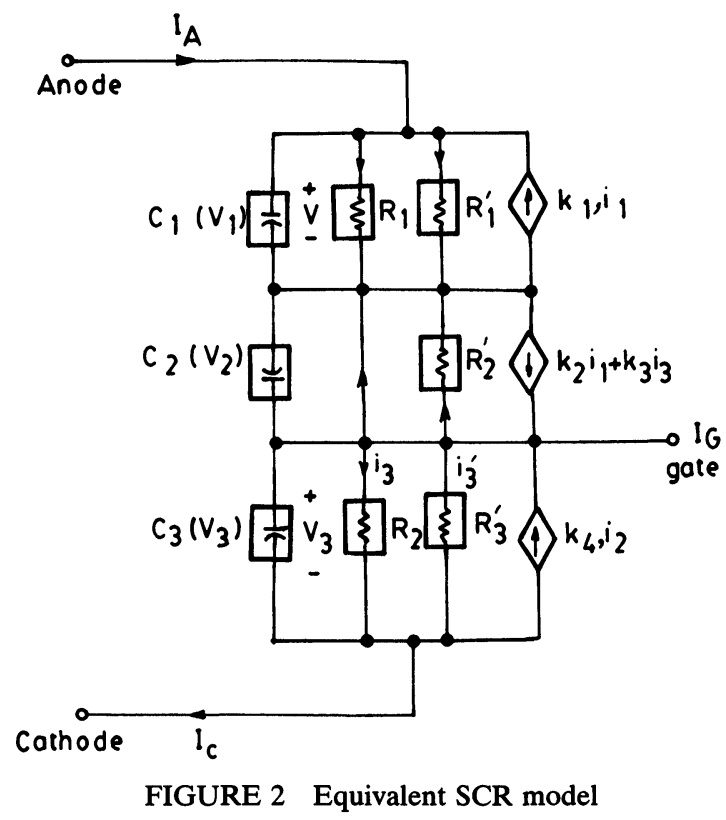

$\mathrm{I}_{\mathrm{S} 11}, \mathrm{I}_{\mathrm{S} 12}, \mathrm{I}_{\mathrm{S} 13}, \mathrm{I}_{\mathrm{S} 14}, \mathrm{I}_{\mathrm{S} 15}, \mathrm{I}_{\mathrm{S} 16},\left(10^{-6} \mathrm{~A}<\mathrm{I}_{\mathrm{sij}}<10^{-10} \mathrm{~A}\right)$ (ideal saturation current component).

$I_{s 21}, I_{s 22}, I_{s 23}$ (nonlinear saturation current components)

$10^{-13} \mathrm{~A}<\mathrm{I}_{\mathrm{s} 2 \mathrm{j}}<10^{-7} \mathrm{~A}$

$\mathrm{V}_{1}, \mathrm{~V}_{2}$ (recombination factor for current components) $\left(0.001<\mathrm{v}_{\mathrm{j}}<1\right)$

(c) AC parameters:

$\mathrm{C}_{01}, \mathrm{C}_{02}, \mathrm{C}_{03}$ (junction capacitance coefficients) $\left(0.5 \mathrm{pf}<\mathrm{C}_{\mathrm{oj}}<4 \mathrm{pf}\right)$

$\mathrm{m}_{1}, \mathrm{~m}_{2} \mathrm{~m}_{3}$ (junction grading coefficients, $0.3<\mathrm{mj}<0.7$ )

$\tau_{1}, \tau_{2}, \tau_{3}$ (minority carrier lifetimes, typically, $0.1 \mathrm{~ns}<\tau \mathrm{j}<20 \mathrm{~ns}$

Nonlinear voltage controlled current sources can be partitioned into non-linear resistors and linear current sources resulting in the model structure shown in Fig. 2.

Non-linear Resistors

$$
\begin{aligned}
& R_{1}: i_{1}=I_{1}\left(e^{V_{1} / V_{T}}-1\right) I_{1}=\left(1-\gamma_{1}\right) I_{S 11}+I_{S 15} \\
& R_{1}^{\prime}: i_{1}^{\prime}=I_{1}^{\prime}\left(e^{V_{1} / 2 V_{T}}-1\right) I_{1}^{\prime}=I_{S 21} \\
& R_{2}: i_{2}=T_{2}\left(e^{\mathrm{V}_{2} / V_{t}}-1\right) T_{2}=\left(1+\gamma_{1}\right) T_{S 12}+(1+\gamma) I_{S 14}
\end{aligned}
$$


$\mathbf{R}_{2}^{\prime}: \mathrm{i}_{2}^{\prime}=\mathrm{I}_{2}^{\prime}\left(\mathrm{e}^{\mathrm{V}_{2} / 2 \mathrm{~V}_{\mathrm{T}}}-1\right) \mathrm{I}_{2}^{\prime}=\mathrm{I}_{\mathrm{S} 22}$

$R_{3}: i_{3}=I_{3}\left(e^{V_{3} / V_{T}}-1\right) I_{3}=\left(1+\gamma_{2}\right) I_{S 13}+I_{S 16}$

$R_{3}^{\prime}: i_{3}^{\prime}=I_{3}^{\prime}\left(e^{V_{3} / 2 V_{T}}-1\right) I_{3}^{\prime}=I_{S 23}$

Coefficient for the first controlled source, $\mathrm{k}_{1}=$

$\mathrm{I}_{\mathrm{S} 12} / \mathrm{I}_{2}$

$\mathrm{k}_{2}=\mathrm{I}_{\mathrm{S} 11} / \mathrm{I}_{1}, \mathrm{k}_{3}=\mathrm{I}_{\mathrm{S} 13} / \mathrm{I}_{3}$

Coefficient for the third controlled source:

$\mathbf{k}_{4}=\frac{\mathrm{I}_{\mathrm{S} 14}}{\mathrm{I}_{2}}$

Equivalence of the two model structure can be seen by putting these equations into KCL equation for Fig. 3(c) and comparing them with the corresponding KCL equation for Fig. 2.

The present SCR model represents a current controlled R-dynamic 2-port characterised as follows:

State Equations:

$\frac{\mathrm{dv}_{1}}{\mathrm{dt}}=\frac{\mathrm{I}_{\mathrm{A}}-\mathrm{f}_{\mathrm{A}}\left(\mathrm{V}_{1}, \mathrm{~V}_{2}\right)}{\mathrm{C}_{1}\left(\mathrm{~V}_{1}\right)}=\mathrm{f}_{1}\left(\mathrm{v}_{1}, \mathrm{v}_{2}, \mathrm{v}_{3}, \mathrm{I}_{\mathrm{A}}, \mathrm{I}_{\mathrm{G}}\right)$

$\frac{d V_{2}}{d t}=\frac{I_{A}-f_{B}\left(V_{1}, V_{2}, V_{3}\right)}{C_{2}\left(V_{2}\right)}=f_{2}\left(V_{1}, V_{2}, V_{3}, I_{A}, I_{G}\right)$

$\frac{d V_{3}}{d t}=\frac{I_{A}+I_{G}-f_{c}\left(V_{2}, V_{3}\right)}{C_{3}\left(V_{3}\right)}=f_{3}\left(V_{1}, V_{2}, V_{3}, I_{A}, I_{G}\right)$

Output equations:

$\mathrm{V}_{\mathrm{ac}}=\mathrm{V}_{1}+\mathrm{V}_{2}+\mathrm{V}_{3}=\mathrm{g}_{1}\left(\mathrm{~V}_{1}, \mathrm{~V}_{2}, \mathrm{~V}_{3}\right)$

$\mathrm{V}_{\mathrm{G}}=\mathrm{V}_{3}=\mathrm{g}_{2}\left(\mathrm{~V}_{1}, \mathrm{~V}_{2}, \mathrm{~V}_{3}\right)$

Derivation of the SCR Circuit Model

Let us consider a 1-dimensional p-n-pn SCR structure. The doping concentration is also shown. If we apply a forward biased voltage $\mathrm{V}_{\mathrm{AC}}$ across the device, the mobile carriers will be depleted from the junctions leaving the depletion layers as shown. 


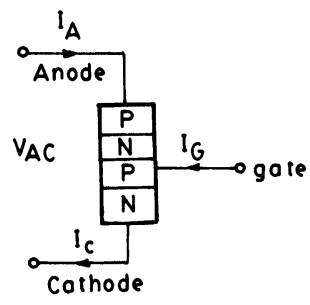

(a) SCR

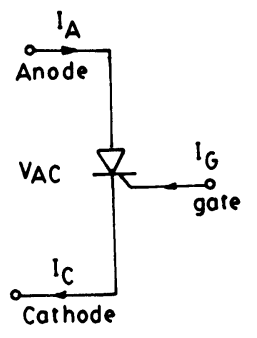

(b) SCR symbol

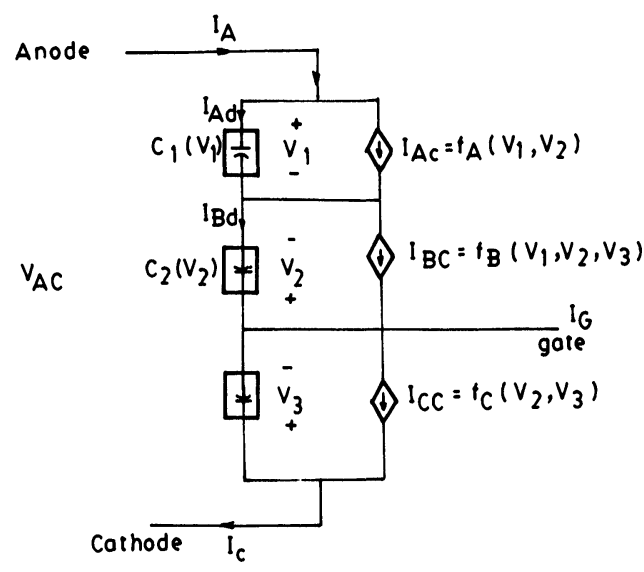

(c) Lumped circuit model for SCR

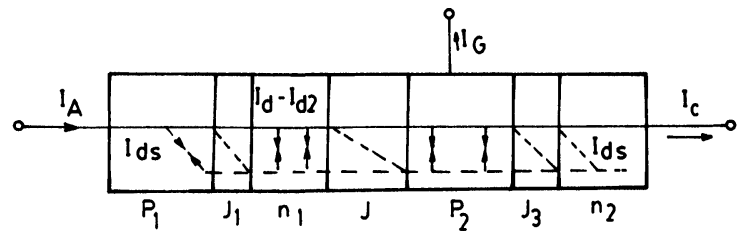

(d) Symbolic representation of diffusion and recombination current components inside and outside of the depletion region

\section{FIGURE 3}

The junctions $\mathrm{J}_{1}$ and $\mathrm{J}_{3}$ will be forward biased while the middle junction will be reverse biased $\left(\mathrm{J}_{2}\right)$.

The Anode current $I_{A}$ is composed of a displacement current $I_{A d}$ and a carrier current $\mathrm{I}_{\mathrm{AC}}$ made up of hole and electron currents injected from junction 1.

$\mathrm{I}_{\mathrm{A}}=\mathrm{I}_{\mathrm{Ad}}+\mathrm{I}_{\mathrm{AC}}=\mathrm{I}_{\mathrm{Ad}}+\left(\mathrm{I}_{\mathrm{An}}+\mathrm{I}_{\mathrm{AC}}\right)$

$I_{A n}$ and $I_{A c}$ denote the hole and $e^{-}$concentrations. The cathode current $I_{c}$ is composed of a displacement current $I_{c d}$ and a carrier current $I_{c c}$ made up of hole and electron injection from junction 3.

$I_{c}=I_{c d}+I_{c c}=I_{c d}+\left(I_{c h}+I_{c e}\right)$ 
The three displacement currents $I_{A d}, I_{B d}$, and $I_{c d}$ are governed by well-known laws between the charge and voltage across the depletion layer and can be modelled by 3 non-linear junction capacitors whose incremental capacitances $\mathrm{C}_{1}\left(\mathrm{v}_{1}\right), \mathrm{C}_{2}\left(\mathrm{v}_{2}\right)$ and $\mathrm{C}_{3}\left(\mathrm{v}_{3}\right)$ are defined by

$c_{j}\left(v_{j}\right)=c_{o j}\left(-v_{j}\right)^{-(1 / m j)}+\frac{I_{s i j j}{ }^{T j}}{v_{T}} e^{v_{j} / v_{T}}$

The carrier currents $\mathrm{I}_{\mathrm{Ac}}, \mathrm{I}_{\mathrm{Bc}}$ and $\mathrm{I}_{\mathrm{cc}}$ are made up of a diffusion current component and a generation recombination current component in the depletion region (see Fig. 3d).

These components are defined below along with their relationships in terms of the junction voltage

(a) Diffusion current components:

$I_{d 1}=I_{S 11}\left(e^{V_{1} / V_{T}}-1\right)=$ hole injected from $J_{1}$. into $\mathrm{N}_{1}$ and which diffused across $\mathrm{N}_{1}$

$I_{d 2}=I_{S 12}\left(e^{V_{2} / v_{T}}-1\right)=$ holes injected from $J_{2}$. into $\mathrm{N}_{1}$ and which diffused across $\mathrm{N}_{1}$.

$I_{d 3}=I_{S 13}\left(e^{V_{3} / V_{T}}-1\right)=$ electron injected from $J_{3}$. into $P_{2}$ and which diffused across $P_{2}$.

$I_{d 4}=I_{S 14}\left(e^{V_{2} / V_{T}}-1\right)=$ Electron injected from $J_{2}$. into $P_{2}$ and which diffused across $P_{2}$.

$I_{d 5}=I_{S 15}\left(e^{V_{1} / V_{T}}-1\right)=$ Electrons injected from $J_{1}$. into $P_{1}$ and which diffused across $P_{1}$.

$I_{d 6}=I_{S 16}\left(e^{V_{3} / V_{T}}-1\right)=$ holes injected from $J_{3}$. into $\mathrm{N}_{2}$ and which diffused across $\mathrm{N}_{2}$.

The parameters $I_{s i j}(j=1$ to 6$)$ are constants that depend on the intrinsic parameters of the device.

(b) Recombination current components outside the depletion region:

$I_{\gamma o 1}=\gamma_{1} I_{d 1}=$ fraction of holes injected from $J_{1}$, which recombine with electrons in $\mathrm{N}_{1}$.

$I_{\gamma 02}=\gamma_{1} I_{d 2}=$ fraction of holes injected from $J_{2}$, which recombine with electrons in $\mathrm{N}_{1}$.

$I_{\gamma 03}=\gamma_{2} I_{d 3}=$ fraction of electrons injected from $J_{3}$, which recombine with holes in $\mathrm{P}_{2}$. 
$I_{\gamma o 4}=\gamma_{2} I_{d 4}=$ fraction of electrons injected from $J_{2}$, which recombine with holes in $\mathrm{P}_{2}$.

The parameters $\gamma_{1}$ and $\gamma_{2}$ are the minority carrier recombination coefficients in $\mathrm{N}_{1}$ and $\mathrm{p}_{2}$, respectively

(c) Recombination-current components inside the depletion region:

$\mathrm{I}_{\gamma \mathrm{i} 1}=\mathrm{I}_{\mathrm{s} 21}\left(\mathrm{e}^{\mathrm{V} 1 / 2 \mathrm{VT}}-1\right)=$ current component due to holes,

which recombine with electrons in $J_{1}$.

$\mathrm{I}_{\gamma \mathrm{i} 2}=\mathrm{I}_{\mathrm{s} 22}\left(\mathrm{e}^{\mathrm{V} 2 / 2 \mathrm{VT}}-1\right)=$ current component due to holes,

which recombine with electrons in $\mathrm{J}_{2}$.

$\mathrm{I}_{\gamma \mathrm{i3}}=\mathrm{I}_{\mathrm{s} 23}\left(\mathrm{e}^{\mathrm{V} 2 / 2 \mathrm{VT}}-1\right)=$ current component due to electrons, which recombine with holes in $\mathrm{J}_{3}$.

The parameters $I_{s 21}, I_{s 22}, I_{s 23}$ are constants, which depend on the intrinsic parameters of the device.

A factor of 2 is an in-added feature of these recombinations currents. The factor 2 corresponds to the fact that while the whole region outside the depletion layer contains only majority carriers, the region inside the depletion layer contains both majority and minority carriers in nearly equal numbers.

Referring to Fig. (3d), we obtain $\mathrm{I}_{\mathrm{Ah}}=$ (hole current injected through $\mathrm{J}_{1}$ into $\mathrm{N}_{1}$ and which diffuses across $\mathrm{N}_{1}$ and collected at $\mathrm{J}_{2}$ ) + (hole current injected through junction 1 into $\mathrm{N}_{1}$ and which reconbined with $\mathrm{e}^{-}$in $\mathrm{N}_{1}$ ) + (hole current which recombined with $\mathrm{e}^{-}$in $\mathrm{J}_{1}$ )-(hole current injected through $\mathrm{J}_{2}$ into $\mathrm{N}_{1}$ and which diffuses across $N_{1}$ and collected at $J_{1}$ ).

$$
\begin{aligned}
& =I_{d 1}+I \gamma_{o 1}+I_{\gamma 21}-I_{d 2}=I_{S 11}\left(e^{V_{1} / V_{T}}-1\right)+V_{1} I_{S 11}\left(e^{V_{1} / V_{T}}-1\right)+ \\
& I_{S 21}\left(e^{V_{1} / 2 V_{T}}-1\right)-I_{S 12}\left(e^{V_{2} / V_{T}}-1\right)
\end{aligned}
$$

IAC $=$ Electron current injected through junction 1 into

$\mathrm{P}_{1}=\mathrm{I}_{\mathrm{d} 3}=\mathrm{I}_{\mathrm{S} 15}-\left(\mathrm{e}^{\mathrm{V}_{\mathrm{I}} / \mathrm{V}_{\mathrm{T}}}-1\right)$

$I_{B C}=$ (hole current injected from $J_{2}$ into $P_{2}+\left(\right.$ current generated in $\left.J_{2}\right)+($ electron current injected from $\mathrm{J}_{2}$ into $\mathrm{N}_{1}$ )

$$
\begin{aligned}
& \left.=I_{d 1}-I_{d 2}-I_{\gamma 02}-I_{\gamma i 2}\right)+\left(I_{\gamma i 2}\right)+\left(I_{d 3}-I_{d 4}-I_{\gamma 04}-I_{\gamma i 2}\right) \\
& =I_{d 1}-I_{d 2}-I_{\gamma 02}+I_{d 3}-I_{d 4}-I_{\gamma i 2}-I_{\gamma 04} \\
& =I_{S 11}\left(e^{V_{1} / V_{\mathrm{T}}}-1\right)-I_{S 21}\left(e^{V_{2} / V_{\mathrm{T}}}-1\right)-\gamma_{1} I_{S 12}\left(e^{V_{2} / V_{T}}-1\right) \\
& +I_{S 13}\left(e^{V_{3}-V_{\mathrm{T}}}-1\right)-I_{S 14}\left(e^{V_{2} / V_{\mathrm{T}}}-1\right)-I_{S 23}\left(e^{V_{2} / 2 V_{\mathrm{T}}}-1\right) \\
& -\gamma_{2} I_{S 14}\left(e^{V_{2} / V_{\mathrm{T}}}-1\right)
\end{aligned}
$$

$I_{c c}=$ (electron current injected through $J_{3}$ into $P_{2}$ and which diffused across $P_{2}$ and collected at $\left.\mathrm{J}_{2}\right)+\left(\right.$ electron current injected through $\mathrm{J}_{3}$ into $\mathrm{P}_{2}$ and which 
recombined with holes in $\left.\mathrm{P}_{2}\right)+\left(\right.$ electron current injected through junction 2 into $\mathrm{P}_{2}$ and which infused across $P_{2}$ and collected at $J_{3}$ )

$$
\begin{aligned}
& =I_{d 3}+I_{v 03}+I_{v i 3}-I_{d 4}=I_{S 13}\left(e^{V_{3} / V_{T}}-1\right)+V_{2} I_{S 13}\left(e^{V_{3} V_{T}}-1\right)+I_{S 23}\left(e^{V_{3} / 2 V_{T}}-1\right) \\
& -I_{S 14}\left(e^{V_{2} / V_{T}}-1\right) \\
& \text { Ich = (hole current injected from junction } \left.3 \text { into } N_{2}\right)=I_{d 6}=I_{s 16}\left(e^{V 3 / V T}-1\right)
\end{aligned}
$$

Substituting equations (40) (41) into equation (38a) and equation (42) into (38b), equation (43), (44) into equation (39), we obtain the element characteristic specified in equations (21-24)

\section{A CIRCUIT MODEL FOR P-N JUNCTION DIODES USING MEMRISTOR-THE MISSING ELEMENT}

\section{The Memristor}

The memristor is a 2-terminal circuit element defined by $U_{m}=R_{m}\left(q_{m}\right) f_{m}$, where $R_{m}\left(q_{m}\right)$ is a linear resistance whose value depends on the charge $q_{m}$ passing through its terminals.

A memristor can be considered therefore as a charge controlled linear resistor.

$R_{m}\left(q_{m}\right)=1 / A \int_{0}^{w_{n}} \frac{d x}{\left(x, q_{m}\right)}$

$\left(x, q_{m}\right)=q \mu_{n} n_{n_{o}}+q \mu_{p}\left|P_{n_{o}}+\frac{q_{m}}{A q L_{p}}\right| \frac{\operatorname{Sinh}\left(W_{n} / L_{p}\right)}{\operatorname{Cosh}\left(W_{n} / L_{p}\right)-1}|x+x| \operatorname{Cosh}\left(x / L_{p}\right)-$

$\operatorname{Coth}\left(\mathrm{W}_{\mathrm{n}} / \mathrm{L}_{\mathrm{p}}\right) \operatorname{Sin} \mathrm{h}\left(\mathrm{X} / \mathrm{L}_{\mathrm{p}}\right) \mid$

$\mu_{\mathrm{n}}=$ electron mobility

$\mu_{\mathrm{p}}=$ hole mobility

$A=$ junction area of the diode

$\mathrm{W}_{\mathrm{n}}=$ width of $\mathrm{n}$-type region (base width)

$\tau_{\mathrm{p}}=$ hole recombination time

$n_{n_{0}}=$ equilibrium electron concentration in n-type region

$P_{n_{0}}=$ equilibrium hole concentration in n-type region 
$L_{p}=\left(D_{p} \tau_{p}\right)^{1 / 2}-$ hole diffusion length

$D_{p}=$ hole diffusion constant.

The controlled current source: $i_{2}=I_{2}\left(q_{m}\right)$

$\mathrm{i}_{2}\left(\mathrm{q}_{\mathrm{m}}\right)=\mathrm{q}_{\mathrm{m}} / \tau$

$\tau=\tau_{\mathrm{p}}\left|1-\operatorname{Sech}\left(\mathrm{W}_{\mathrm{n}} / \mathrm{L}_{\mathrm{p}}\right)\right|$

$\tau \rightarrow$ effective hole life time

The controlled current source

$$
\begin{aligned}
& i_{1}=I_{1}\left(i, i_{j}, v_{j}, q_{m}\right)=I_{1 f} \mu(i)+I_{1 \gamma} \mu(-i) I_{1 f}=I_{s}\left[\exp \left(\frac{V_{j}}{V_{T}}\right)-1\right]+\frac{C_{d}\left(V_{j}\right)}{C_{j}\left(V_{j}\right)} i_{j} \\
& c_{d}\left(V_{j}\right)=\frac{I_{6} \tau}{V_{T}} \exp \left(\frac{V_{i}}{V_{T}}\right) \\
& I_{1 \gamma}=i-c_{j}\left(V_{j}\right) \max \left(V_{a}, V_{b}\right) \\
& \gamma_{a}=\frac{C_{j}\left(V_{j}\right)\left\{1+\frac{\left|q_{m}\right|+I_{s} \tau}{\left(|i|+I_{s}\right) \tau} \mu\left(-V_{j}\right)\right\}}{\gamma_{b}=-\max \left(\gamma_{c}, \gamma_{d}\right)} \\
& \gamma_{c}=\frac{I_{s}\left[\exp \left(\frac{V_{j}}{V_{T}}\right)-1\right]-i}{C_{d}\left(V_{j}\right)+C_{j}\left(V_{j}\right)} \\
& \gamma_{d}=\frac{V_{n o}\left|1+\exp \left(\frac{V_{T} \operatorname{Sinh}\left(W_{n} / L_{p}\right)}{1-0.5 V_{j} \mu\left(-v_{j}\right) V_{T}}\right)\right|\left\{0.25 \frac{\left|q_{m}\right|+I_{s} \tau}{|i|+(s) \tau} \mid\right.}{A q L_{p}\left[\operatorname{Cosh}\left(W_{n} / L_{p}\right)-1\right]} \mid q_{m}
\end{aligned}
$$

The Memristive diode model is prescribed by $\mathrm{p}$ physical parameter $\left\{\psi_{\mathrm{o}}, \in, \mathrm{N}_{\mathrm{D}}, \mu_{\mathrm{n}}, \mu_{\mathrm{p}}, \eta_{\mathrm{n}_{\mathrm{o}}}\right.$, pno, $\left.\mathrm{A}, \mathrm{W}_{\mathrm{n}}, \mathrm{D}_{\mathrm{p}}, \tau_{\mathrm{p}}, \mathrm{V}_{\tau}, \mathrm{I}_{\mathrm{s}}\right\}$ and two empirical parameters $\{\alpha, \beta\}$.

\section{Dynamic behavior of junction diodes during reverse transients}

Let us consider the diode circuit shown in Fig. 4a. The switch $S$ is thrown from right to left at $t=t_{o}$ and before to the diode is in steady state with a current $i(t)=I_{F}$, and a voltage $v(t)=E_{f}$. Reverse transient waveform is shown in Fig 4(a) and (b). 


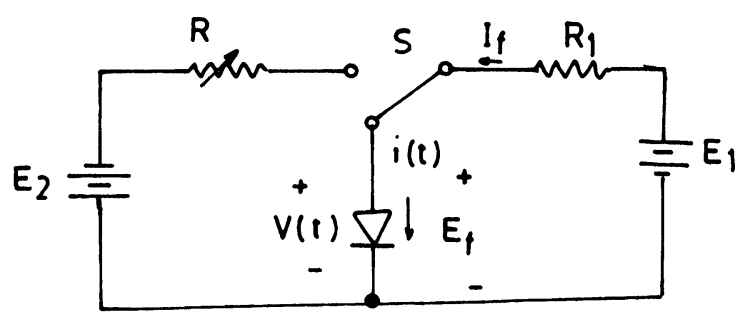

(a) Circuit for measuring reverse transmit

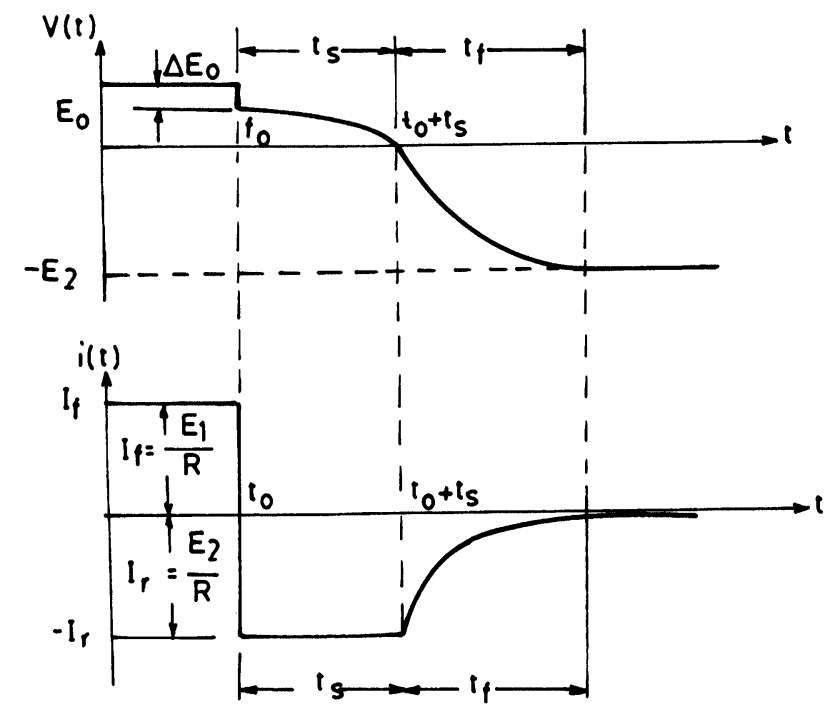

(b) Voltage and current waveforms during reverse transmit

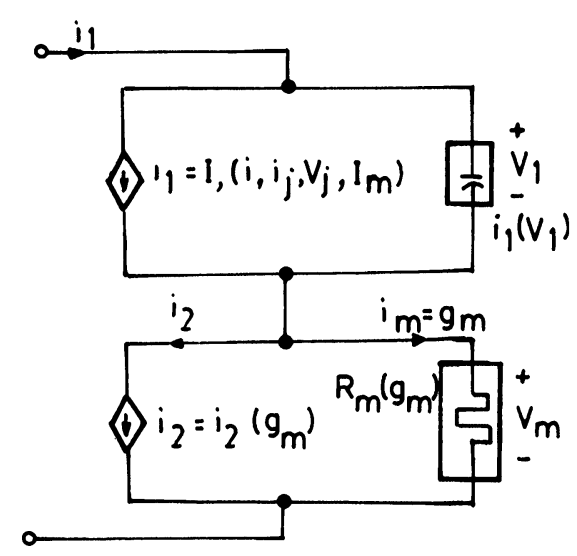

(c) Memristive model for junction divide

FIGURE 4 

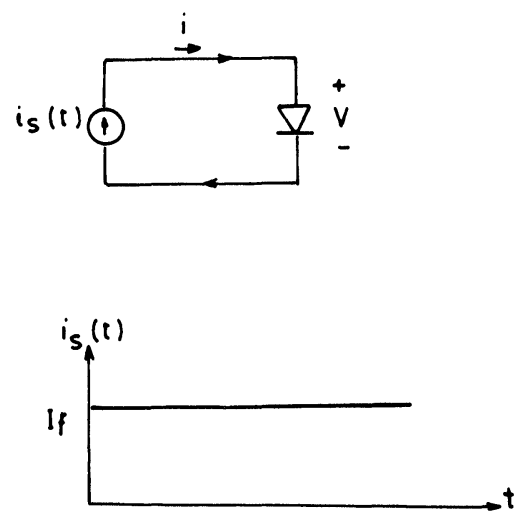

FIGURE 5

Dynamic behavior of junction diodes during forward transient

Let us consider circuit in Fig 5 . A positive step current $I_{s}(t)$ is applied at $t=0$ when the diode is in zero state. The associated voltage wave-form $v(t)$ for $t>0$ is shown in Fig 6(b) for three values of $I_{f}$.

\section{Derivation of the Model}

Let us consider a p-n junction $\left(\mathrm{N}_{\mathrm{A}}>>\mathrm{N}_{\mathrm{D}}\right.$, step junction).

$C_{j}$ is used to represent effective transition layer capacitance. $i_{1}$ is used to simulate the leakage carriers through the layer. $R_{m}$ is used to simulate the conductance modulation of neutral region $i_{2}$ is used to represent the recombination of carriers

$\underline{\text { Non-linear junction capacitance } C_{j}\left(V_{j}\right)}$

$$
\mathrm{C}_{\mathrm{j}}\left(\mathrm{V}_{\mathrm{j}}\right)=\frac{\mathrm{K}_{\mathrm{a}}}{2}\left(\psi_{\mathrm{o}}-\mathrm{V}_{\mathrm{j}}\right)^{-1 / 2}
$$

$\mathrm{K}_{\mathrm{a}}=\mathrm{A}\left(2 \in \mathrm{q} \mathrm{N}_{\mathrm{D}}\right)^{1 / 2}$

Memristor $\mathbf{R}_{\mathrm{m}}\left(\mathrm{q}_{\mathrm{m}}\right)$

$\mathrm{N}_{\mathrm{A}}>>\mathrm{N}_{\mathrm{D}}$ so conductance of p-region is much greater than that of n-type, i.e., resistance of diode is mainly contributed by the n-type region (the base region), conductivity $\sigma(x)$ of base region is given by

$\sigma(\mathbf{x})=\left|\mu_{\mathrm{n}} \mathrm{n}_{\mathrm{xo}}+\mu_{\mathrm{p}} \mathrm{p}_{\mathrm{n}}(\mathrm{x})\right|$

Let us consider the one dimensional steady-state diffusion equation

$\mu_{\mathrm{s}}^{2} \frac{\mathrm{p}_{\mathrm{n}}^{\prime}(\mathrm{x})}{\alpha \mathrm{x}^{2}}-\frac{\mathrm{P}_{\mathrm{n}}^{\prime}(\mathrm{x})}{\mathrm{L} \mathrm{p}^{2}}=\mathrm{O}, \mathrm{P}_{\mathrm{n}}^{\prime}(\mathrm{x})=\mathrm{P}_{\mathrm{n}}(\eta)$ - pno excess hole concentration in n-type region. 

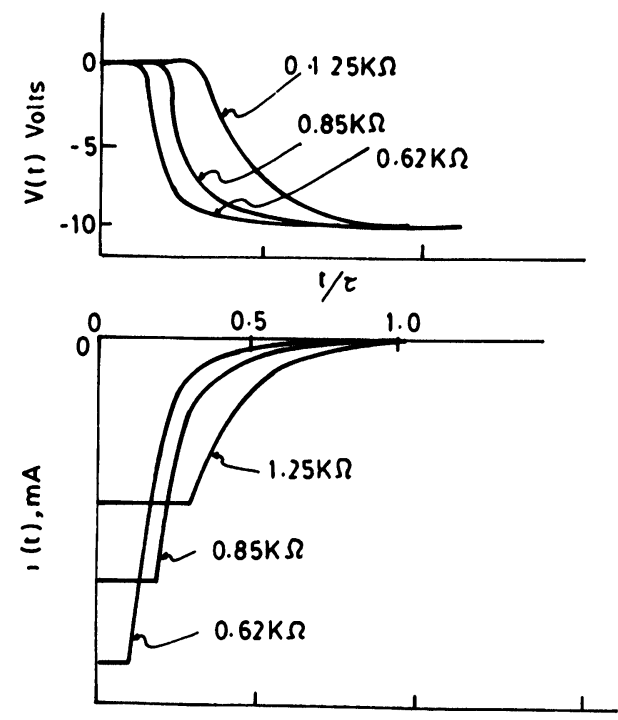

(a) Reverse transmit

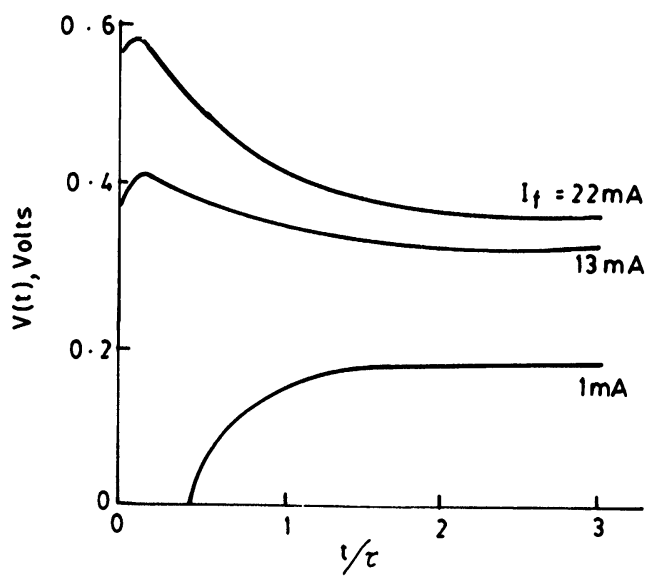

(b) Forward transmit FIGURE 6

Applying boundary condition

(a) At $\mathrm{x}=0$

$P_{n}^{\prime}(x)=P_{n}^{\prime}(0)-P_{n o}=P_{n o} \exp \left(V_{j} / V_{T}\right)-P_{n o}$

$=P_{n o}\left|\exp \left(\frac{V_{j}}{V_{T}}\right)-1\right|$ (law of junction)

$\mathrm{V}_{\mathrm{j}} \rightarrow$ applied voltage across the junction diode. 
(b) at $\mathrm{x}=\mathrm{Wx}$

$\mathrm{s}_{\mathrm{p}} \mathrm{xP}_{\mathrm{x}}^{\prime}\left(\mathrm{W}_{\mathrm{x}}\right)=\frac{1}{\mathrm{q}} \mathrm{J}_{\mathrm{p}}\left(\mathrm{W}_{\mathrm{n}}\right)=-\left.\mathrm{D}_{\mathrm{p}} \frac{\alpha \mathrm{P}_{\mathrm{n}}^{\prime}(\mathrm{x})}{\alpha \mathrm{x}}\right|_{\text {Xown }}$

$s_{p} \rightarrow$ Recombination velocity of holes

For large $S_{p}^{\prime}$ solution of steady state diffusion, the equation is

$P_{n}^{\prime}(x)=P_{n}^{\prime}(0)\left|\cosh \left(\frac{X}{L_{p}}\right)-\operatorname{Coth}\left(\frac{W_{n}}{L_{p}}\right) \operatorname{Sinh}\left(\frac{X}{L_{p}}\right)\right|$

The stored excess minority charge is

$$
\begin{aligned}
& \mathrm{q}_{\mathrm{p}}^{\prime}=\int_{0}^{\mathrm{W}_{\mathrm{n}}} \operatorname{Aq} \mathrm{P}_{\mathrm{n}}^{\prime}(\mathrm{x}) \mathrm{dx}=\operatorname{AqP} \mathrm{P}_{\mathrm{n}}^{\prime}(\mathrm{o}) \mathrm{L}_{\mathrm{p}}\left\{\frac{\operatorname{Cosh}\left(\mathrm{W}_{\mathrm{n}} / \mathrm{L}_{\mathrm{p}}\right)-1}{\operatorname{Sinh}\left(\mathrm{W}_{\mathrm{n}} / \mathrm{L}_{\mathrm{p}}\right)}\right\} \\
& \Rightarrow \mathrm{P}_{\mathrm{n}}^{\prime}(\mathrm{x})=\frac{\mathrm{q}_{\mathrm{n}}^{\prime}}{\operatorname{AqL} L_{\mathrm{p}}}\left\{\frac{\operatorname{Sinh}\left(\mathrm{W}_{\mathrm{n}} / \mathrm{L}_{\mathrm{p}}\right)}{\operatorname{Cosh}\left(\mathrm{W}_{\mathrm{n}}-1\right)}\left|\operatorname{Cosh}\left(\frac{\mathrm{X}}{\mathrm{L}_{\mathrm{p}}}\right)-\operatorname{Coth}\left(\frac{\mathrm{W}_{\mathrm{n}}}{\mathrm{L}_{\mathrm{p}}}\right) \operatorname{Sinh}\left(\frac{\mathrm{X}}{\mathrm{L}_{\mathrm{p}}}\right)\right|\right.
\end{aligned}
$$

The stored excess minority charge in steady state may be $\mathrm{q}^{\prime}$ but in a non-steady state it is $q_{m}$ when $q_{m}^{\prime} \neq 0 . q_{m}$ explains the conductivity modulation in base region.

$P_{n}^{\prime}(x)=\frac{q^{\prime} p}{A q L_{p}}\left(\frac{\operatorname{Sinh}\left(W_{n} / L_{p}\right)}{\operatorname{Cosh}\left(W_{n} / L_{p}\right.}-1\right)\left[\operatorname{Cosh}\left(\frac{x}{L_{p}}\right)-\operatorname{Coth}\left(\frac{W_{n}}{L_{p}}\right) \operatorname{Sinh}\left(\frac{X}{L_{p}}\right)\right]$

$R_{m}\left(q_{m}\right)=\int_{0}^{w_{n}} \frac{d x}{A_{o}\left(x, q_{m}\right)}$

The controlled current source $i_{2}=I_{2}\left(q_{m}\right)$ from equation (41):

$$
\begin{aligned}
& \mathrm{q}_{\mathrm{p}}^{\prime}=\operatorname{Aq} \mathrm{p}_{\mathrm{n}}^{\prime}(\mathrm{o}) \mathrm{L}_{\mathrm{p}}\left[\frac{\operatorname{Cosh}\left(\mathrm{W}_{\mathrm{n}} / \mathrm{L}_{\mathrm{p}}\right)-1}{\operatorname{Sinh}\left(\mathrm{W}_{\mathrm{n}} / \mathrm{L}_{\mathrm{p}}\right)}\right]=\left[\frac{\operatorname{AqD}_{\mathrm{p}} \mathrm{P}_{\mathrm{n}}^{\prime}(\mathrm{o})}{\mathrm{L}_{\mathrm{p}}} \operatorname{Coth}\left(\frac{\mathrm{W}_{\mathrm{n}}}{\mathrm{L}_{\mathrm{p}}}\right)\right] \\
& {\left[\operatorname{Sech}\left(\frac{\mathrm{W}_{\mathrm{n}}}{\mathrm{L}_{\mathrm{p}}}\right)\right]}
\end{aligned}
$$

where we have used the identity $L_{p}^{2}=D_{p} \tau_{p}$. On the other hand, the diode current is given by $=$

$$
\mathrm{i} \cong \mathrm{a} \mathrm{j}_{\mathrm{p}}(\mathrm{o})=\frac{-\mathrm{AqD}_{\mathrm{p}} \mathrm{jp}_{\mathrm{n}}(\mathrm{x})}{\mathrm{jx}} \mid \mathrm{x}=0
$$


$i=\frac{A q D_{p} j p_{n}^{\prime}(o)}{L_{p}} \operatorname{Coth}\left(\frac{W_{n}}{L_{p}}\right)$

$\mathrm{q}_{\mathrm{p}}^{\prime}=\mathrm{i} \tau$

where $\tau$ is the effective hole life time defined in the equation. We define $i_{2}$ as $\mathrm{i}_{2}=\frac{q_{m}}{\tau}$

At steady state, $\mathrm{q}_{\mathrm{m}}=0 \Rightarrow \mathrm{q}_{\mathrm{p}}^{\prime}=\mathrm{q}_{\mathrm{m}}$

The controlled current source:

$\mathrm{i}_{1}=\mathrm{I}_{1}\left(\mathrm{i}, \mathrm{ij}, \mathrm{V}_{\mathrm{j}}, \mathrm{q}_{\mathrm{m}}\right)$

$i_{1}=\left\{\begin{array}{cc}i_{1 f}, & i>0 \\ i_{1 \gamma} & , i<0\end{array}\right.$

$I_{1 f}=I_{s}\left[\exp \left(\frac{V_{j}}{V_{T}}\right)-1\right]+C_{2}\left(V_{j}\right)\left[\frac{i_{1}}{C_{j}}\left(V_{j}\right)\right]=I_{s}\left[\exp \left(\frac{V_{j}}{V_{T}}\right)-1\right]+$

$C_{d}\left(V_{j}\right) \frac{d V_{j}}{d t}-\frac{d v_{j}}{d t}$

$=\frac{V_{T} P_{n}^{\prime}(o, t)}{P_{n o} \exp \left(\frac{V_{j}}{V_{T}}\right) 0.25 \tau\left[\frac{\left|q_{m}\right| t+I_{s} \tau}{\left(|i(t)|+I_{s}\right) \tau}\right]^{\beta}}=\gamma_{d}$

we postulate $\gamma_{c}$ and $\gamma_{a}$ as

$\gamma_{a}=\frac{i}{C_{j}\left(V_{j}\right)\left\{1+\alpha\left[\frac{\left|q_{m}\right|+I_{s} \tau}{\left(|i|+I_{s}\right) \tau}\right] \mu\left(-V_{j}\right)\right\}}$

$\gamma_{c}=\frac{i_{j}-i}{C_{d}\left(V_{j}\right)+C_{j}\left(V_{j}\right)}=\frac{-d v_{j}}{d t}$

$\operatorname{Max}\left(\gamma_{\mathrm{a}}^{\prime} \gamma_{\mathrm{b}}\right)=\gamma_{\mathrm{b}}$

$\gamma_{b}=-\max \left(\gamma_{c}, \gamma_{d}\right)$

when $\mathrm{V}_{\mathrm{j}}>0$

$\gamma_{b}$ selects between maximum of $\gamma_{c}$ and $\gamma_{d}$. When $V_{j}<0$,

$\gamma_{a}=\frac{i}{c_{j}\left(V_{j}\right)\left\{1+\alpha \frac{\left|q_{m}\right|+I_{s} \tau}{\left(|i|+I_{s}\right) \tau}\right\}}$ 


\section{CONCLUSION AND DISCUSSION OF RESULTS}

(A) For Gunn device Let the given circuit be a 'single cavity' Gunn diode circuit, the cavity can be represented by a parallel resonant circuit combination of $L, R$ and $C$ circuit elements.

a Non-linear $\mathrm{V}(\mathrm{E})$ curve is assumed to be of the form

$V(E)=\frac{8 X 10^{3} E+3.12 \times 10^{-8} E^{4}}{1+3.9 \times 10^{-15} E^{4}}$

subject to the length of sample $\mathrm{L}=10 \mu_{\mathrm{m}}$

The following are the Gunn diode circuit model parameters:

$\mathrm{X}_{\mathrm{o}}=1 \mathrm{X} 10^{16} \mathrm{~cm}^{-3}, \mathrm{~L}=10 \mu_{\mathrm{m}}, \mathrm{A}=1 \mu_{\mathrm{m}}^{2}, \mathrm{C}_{1}=1.16 \times 10^{-7} \mathrm{~F}, \mathrm{C}_{2}=1 \mathrm{~F}$

Using the zero diffusion circuit model, let us find out the behavior of Gunn diode under different steady-state modes of operation, namely, the transit time mode, the delayed domain mode, quenched mode, and LSA mode. Observe the behavior of following circuit variables -

i) Diode terminal voltage $\mathrm{V}(\mathrm{t})$

ii) Diode terminal current $\mathrm{i}(\mathrm{t})$

iii) Excess capacitor voltage $V_{1}(t)$ across $C_{1}$.

iv) Steady state waveform for $V_{2}(t)$ across $C_{2}$.

v) Steady state waveforms for timing capacitor $C_{3}$.

Let $\mathrm{E}_{\mathrm{t}}=4000$ Volt

\section{Transit Time Mode}

Steady state waveform for $\mathrm{V}(\mathrm{t}), \mathrm{V}_{1}(\mathrm{t}), \mathrm{V}_{2}(\mathrm{t})$ and $\mathrm{V}_{3}(\mathrm{t})$ are plotted in Fig. 7 corresponding to following circuit parameters:

$$
\begin{array}{ll}
\mathrm{R}=1 \Omega, & \mathrm{L}=0.5 \times 10^{-10} \mu \mathrm{H} \\
\mathrm{C}=1 \times 10^{-2} \mathrm{~F}, & \mathrm{~V}_{\mathrm{B}}=4.8 \mathrm{~V}
\end{array}
$$

In the transit time mode, $V_{3}(t)$ seems as a time base whose period is equal to the time taken by anode with velocity $V_{D}$. This occurs when $V_{3}(t)$ increases to $A q n_{o} L$ point (1), the resistor $R_{3}$ becomes $\tau d / C_{2}(\tau d=10$ p.s.). The domain capacitor voltage $V$ discharges through $R_{3}$ with time constant $\tau$ d. As $V_{2}$ drops, $V_{1}$ rises to take up the slack until $V_{1}(t)=E_{t} L$ point (2). At this point, $R_{1}\left(V_{1}\right)$ becomes a short circuit and $V_{3}(t)$ drops to zero. This forces $R_{1}\left(V_{1}\right)$ to switch immediately back to an open circuit and $\mathrm{C}_{3}$ begins to charge through the 'time-base' current source $\mathrm{I}_{\mathrm{c}}$. Point (2) correspondes to the nucleation of a new domain at the cathode. As domain voltage $V_{2}(t)$ increases $V_{1}(t)$ decreases (Fig. 4).

At point (3), the high field domain matures into a steady state and $V_{1}(t)$ and $V_{2}(t)$ remain constant as domain traverses towards the anode.

Since $V_{2}(t)>0, R_{2}$ always remains an open circuit. Finally, as the time base voltage $V_{3}(t)$ reaches $(4)$, this is the instant where the domain arrives at the anode and cycle is repeated. 


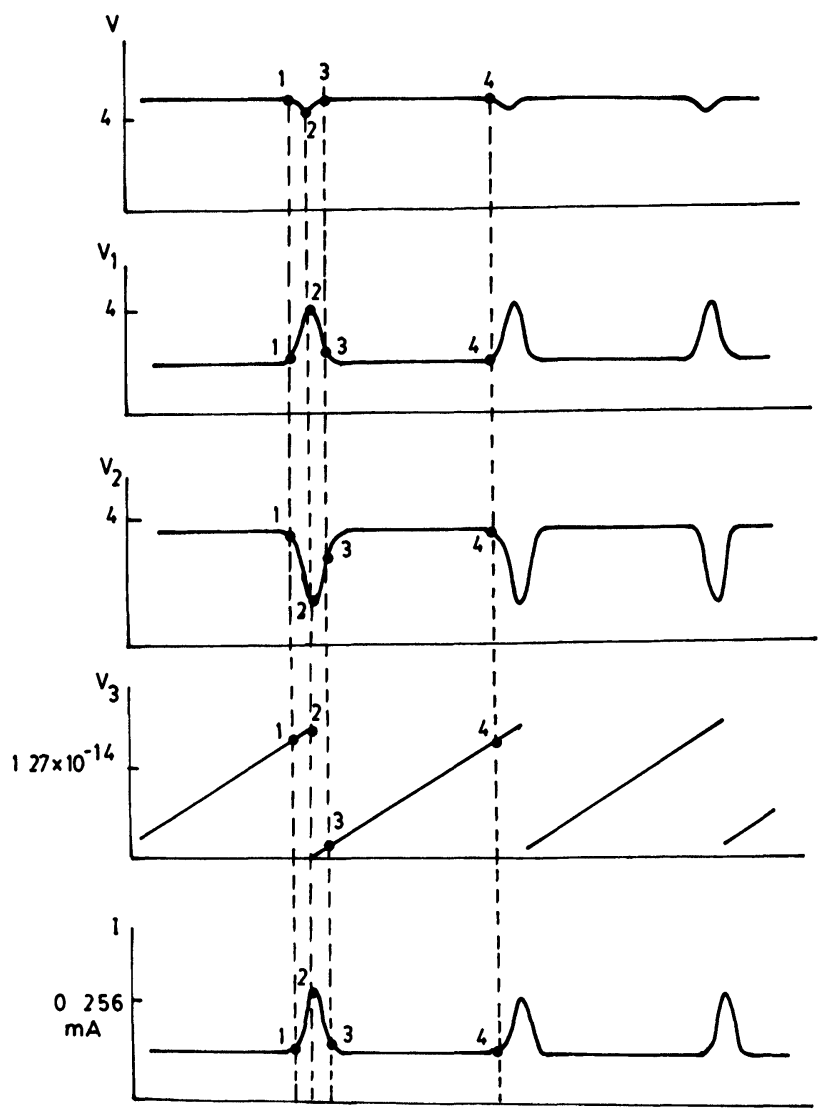

FIGURE 7 Waveforms associated with Gunn-Diode circuit operating under the transit time mode. $\Delta t=57 \times 10^{-12} \mathrm{sec}$.

\section{Delayed Domain Mode}

$\mathrm{V}(\mathrm{t}), \mathrm{I}(\mathrm{t}), \mathrm{V}_{1}(\mathrm{t})$ and $\mathrm{V}_{3}(\mathrm{t})$ are plotted in Figs. 8(a), (b), (c), (d) and (e) respectively. The operation of the delayed domain mode can be illustrated with the help of Fig. 8. Again, let us begin at point (1) on the time-base voltage waveform $V_{3}(t)$ corresponding to the time instant when the domain reaches the anode, thereby switching $R_{3}\left(V_{3}\right)$ to its low-resistance state. Hence, the domain capacitor voltage $V_{2}(t)$ discharges rapidly, through $R_{3}$ until it reaches zero volts (point 3 ), at which time resistor $R_{2}\left(V_{2}\right)$ switches from an open to a short-circuit. From here on the domain remains extinguished while $V_{1}(t)$ takes up whatever slack needed to satisfy $\mathrm{KVL}$ in order to maintain a nearly sinusoidal voltage $\mathrm{V}$ across the highly resonant circuit. As $V_{1}(t)$ increases beyond the threshold voltage $v_{1}=1$ (point 5 ), $R_{1}\left(V_{1}\right)$ switches from a short to an open circuit. A slight increment of time thereafter, $V_{2}$ becomes positive once again since $V$ continues to increase at point (5). This causes $\mathbf{R}_{2}\left(\mathrm{~V}_{2}\right)$ to switch from a short to an open circuit, thereby starting the time-base sawtooth waveform until the domain reaches the anode, and then the cycle repeats itself. 


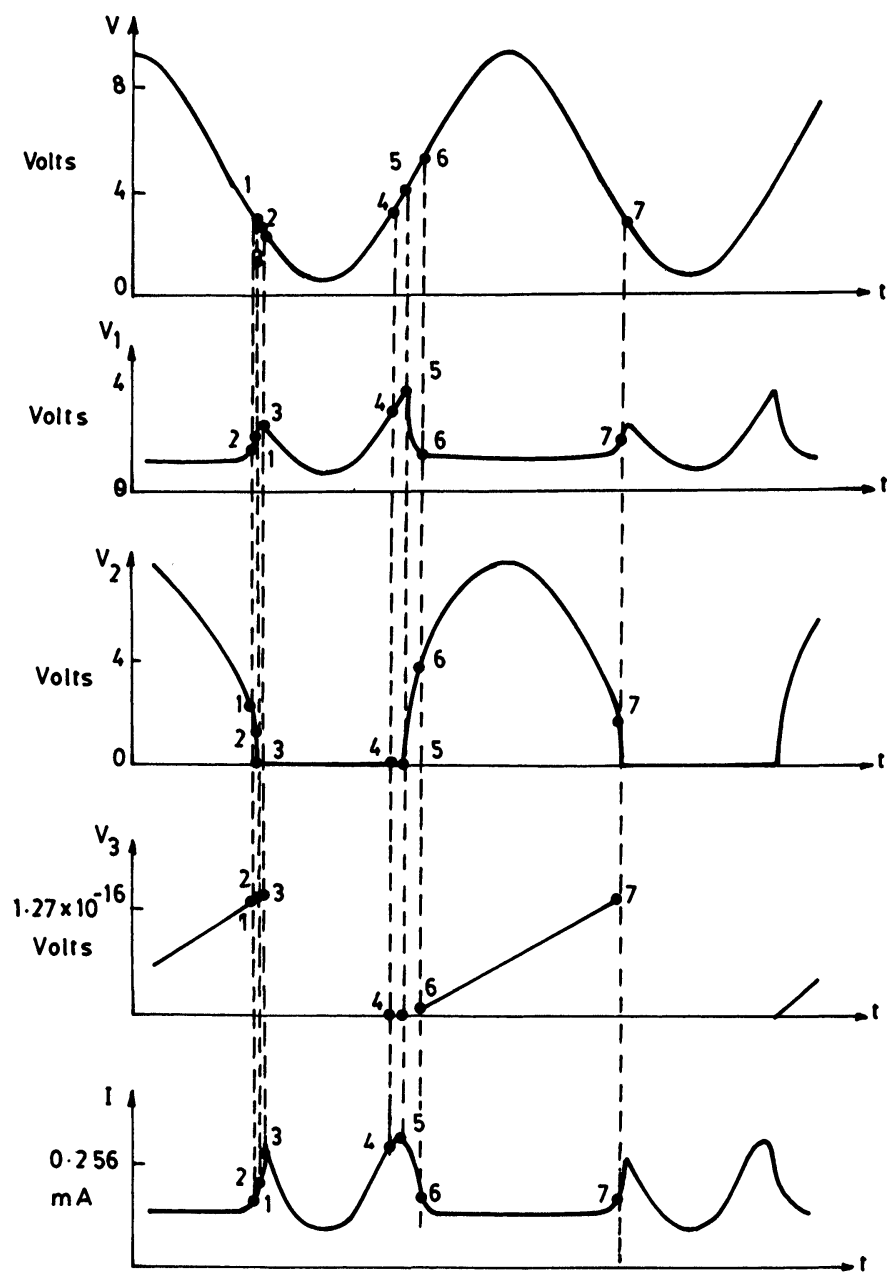

FIGURE 8 Waveforms corresponding to delayed domain mode. $\Delta t m=75 \times 10^{-12} \mathrm{sec}$.

\section{Quenched Domain Mode}

Using the model and circuit parameters given in Fig. 9 for the quenched domain, the steady-state voltage waveforms $V(t), I(t), V_{1}(t), V_{2}(t)$ and $V_{3}(t)$ are shown in Figs. 10 (a), (b), (c), (d) and (e).

The operation of the quenched domain mode can be illustrated with the help of Fig. 10. Unlike the preceding examples, let us start with point (1) in Fig (10.C), where the domain voltage $V_{2}(t)$ drops to zero, thereby switching $R_{2}\left(V_{2}\right)$ to a short circuit. This causes $V_{3}(t)$ to drop instantaneously to zero and remain so until $V_{1}(t)$ rises above the normalized threshold voltage $V_{1}=1$ at point $(4)$, thereby switching $R_{1}\left(V_{1}\right)$ to an open circuit. Thereafter, the domain voltage $V_{2}(t)$ begins to build up while the time-base voltage $V_{3}(t)$ rises to keep track of its motion. In sharp contrast with the operation of the preceding examples, however, observe that the domain voltage $V_{2}(t)$ becomes zero at point (1) before the domain reaches the anode. 


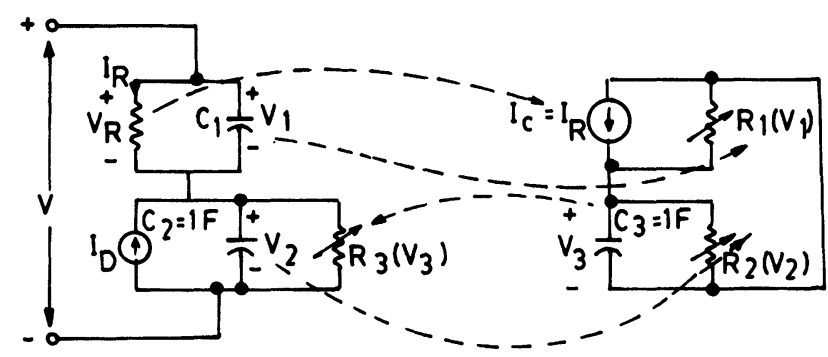

FIGURE 9 Complete Gunn-Diode model for zero diffusion.

Consequently, $R_{3}\left(V_{3}\right)$ remains an open-circuit for all times and the timing circuit therefore plays no role in determining the waveforms in the quenched domain mode. The term 'quench' is used to emphasize that the high-field domain in this case is extinguished by the waveforms $(\mathrm{V}(\mathrm{t}))$ and not by discharging at the anode.

\section{LSA Mode}

The waveforms corresponding to the preceding examples for the LSA mode are shown in Fig. 11. Here we observe that $V_{1}(t)$, because $V_{2}(t)=0$ everywhere except over a very small time interval where $V_{2}(t)$ increases to no longer than 0.01 . Consequently, $R_{2}\left(V_{2}\right)$ becomes a short circuit most of the time, and the domain can be assumed to be almost non-existing. Since the time-base voltage $V_{3}(t)$ is also almost zero, $R_{3}\left(V_{3}\right)$ remains an open circuit for all times in the LSA mode.

(B) For SCR-

Fig. 12 shows a family of $I_{A}$ versus $V_{A}$ curves computed numerically for a typical SCR. They resemble the basic static characteristics.

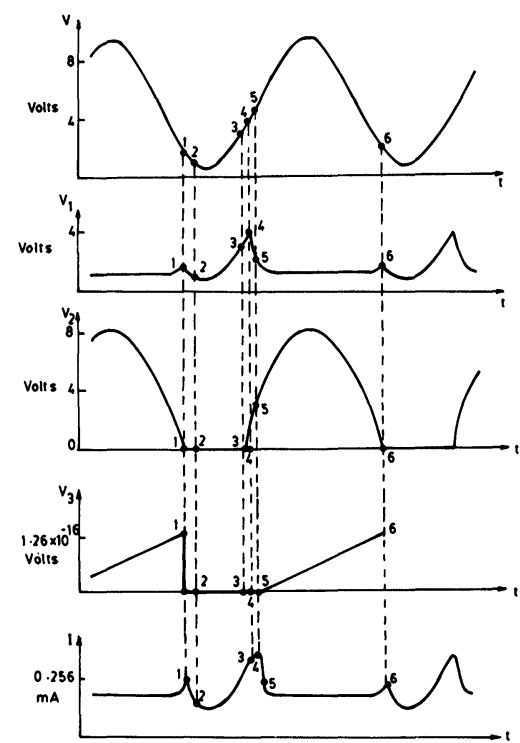

FIGURE 10 Waveforms associated with Gunn-Diode operating under the Quenched Domain mode. 


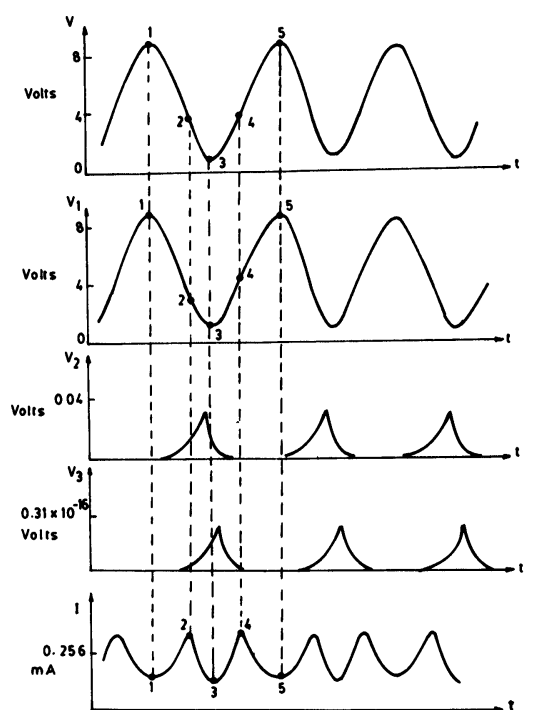

FIGURE 11 Voltage and current waveforms of the Gunn-Diode under the LSA mode. $\Delta t=0.33 \times$ $10^{-10} \mathrm{sec}$.

The dc parameters chosen for this example are as follows:

$$
\begin{aligned}
& I_{s i j}=(i=1)=10^{-11 \mathrm{~A}}(j=1-6) \\
& I_{s 21}=I_{s 22}=I_{s 23}=10^{-7 \mathrm{~A}} \\
& \gamma_{1}=\gamma_{2}=0.01
\end{aligned}
$$

To demonstrate that the internal dynamics of the SCR is much more complicated than that of bipolar transistors, we apply a very low frequency triangular voltage waveform $V_{G}(t)$, as shown in Fig. (13 b), and use the computer to obtain the gate

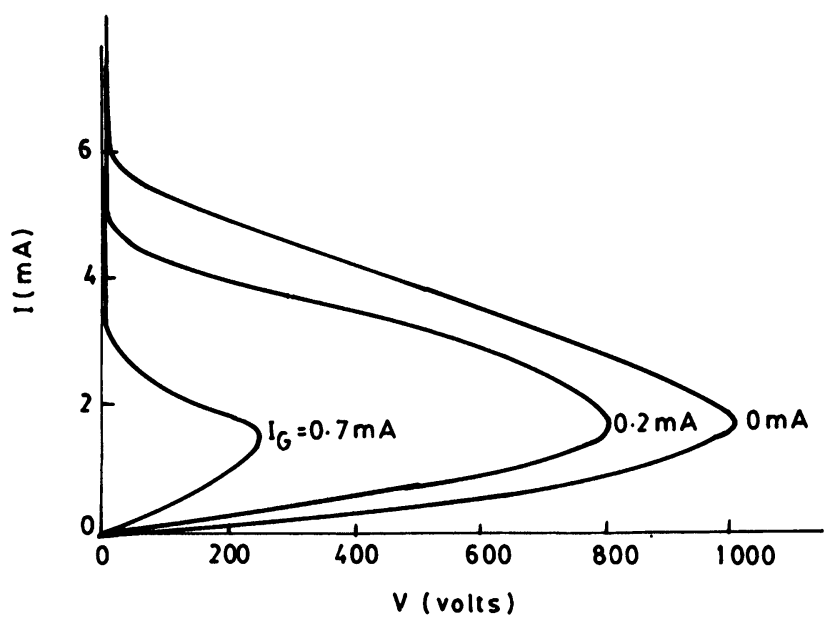

FIGURE 12 Static characteristic of SCR. 
current $I_{G}(t)$ shown in Fig (13 c). The associated $I_{G}$ versus $v_{G}$ relationship is shown in Fig (13 d). Note that it is a multi-valued curve. This multi-value relationship is seen to persist even at extremely low operating frequencies. In other words, the SCR is truly a "dynamic" device. The model parameters chosen for this example are:

a) Physical parameters-

$\psi_{\mathrm{o}}=1, \mathrm{~V}_{\mathrm{T}}=26 \mathrm{mV}$ at $27^{\circ} \mathrm{C}$.

b) DC parameters-

$$
\begin{aligned}
& I_{s i j}=10^{-14} \mathrm{~A}(i=1, j=1 \text { to } 6) \\
& I_{s 21}=I_{s 22}=I_{s 23}=5 \times 10^{-12} \mathrm{~A}: \\
& \gamma_{1}=\gamma_{2}=0.01 \\
& \text { c) AC parameters- } \\
& C_{o 1}=2_{p} F, C_{o 2}=1_{p} F, C_{o 3}=2{ }_{p} F \\
& m_{1}=m_{2}=m_{3}=0.5 \\
& \tau_{1}=1 \mathrm{~ns}, \tau_{2}=10 \mathrm{~ns}, \tau_{3}=1 \mathrm{~ns}
\end{aligned}
$$
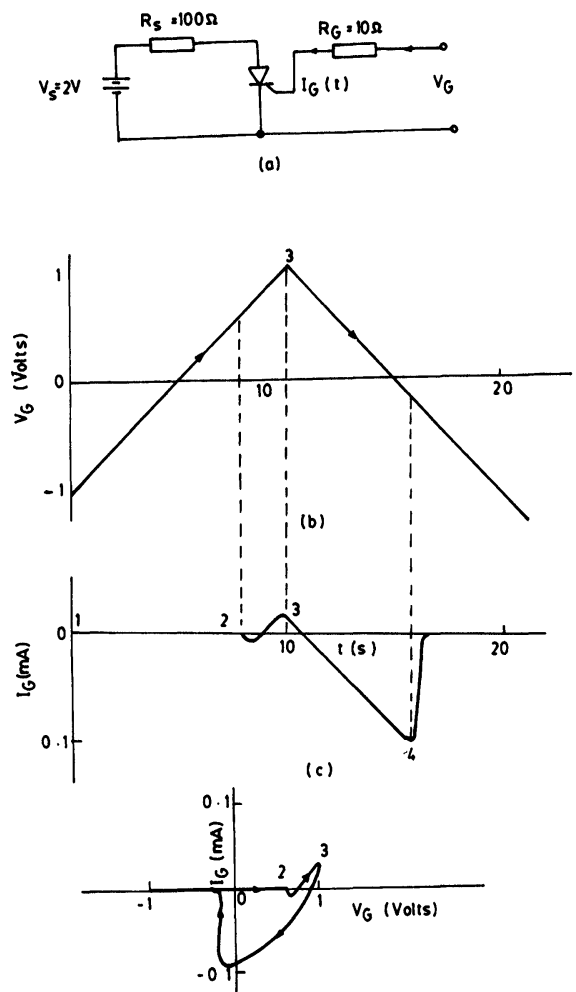

(d)

FIGURE 13 (a): Biased SCR circuit (b): Applied gate voltage Vs(t) (c): Gate current waveform (d): Multivalued $\mathrm{V}_{\mathrm{G}}-\mathrm{I}_{\mathrm{G}}$ relationship. 
(C) For memristor and p-n junction-

Let us consider a silicon diode with the following parameters:

$$
\begin{array}{lc}
\mathrm{N}_{\mathrm{D}}=10^{15} \mathrm{~cm}^{-3} & \tau_{\mathrm{p}}=10^{-7} \mathrm{Sec} \\
\mu_{\mathrm{n}}=1350 \mathrm{~cm}^{2} / \mathrm{V}-\mathrm{Sec} & \mathrm{W}_{\mathrm{n}}=5 \mathrm{~L}_{\mathrm{p}} \\
\mu_{\mathrm{p}}=480 \mathrm{~cm}^{2} / \mathrm{V}-\mathrm{Sec} & \mathrm{T}=300^{\circ} \mathrm{K} \\
\mathrm{I}_{2}=1 / 2 \times 10^{-12} \mathrm{Amp} & \psi_{\mathrm{o}}=0.9 \text { Volt }
\end{array}
$$

From these parameters we obtain,

$$
\begin{aligned}
& \mathrm{D}_{\mathrm{p}}=12.5 \mathrm{~cm}^{2} / \mathrm{Sec} \\
& \mathrm{L}_{\mathrm{p}}=1.12 \times 10^{3} \mathrm{~cm} \\
& \mathrm{n}_{\mathrm{n}}^{\mathrm{o}}=10^{5} \mathrm{~cm}^{-3} \\
& \mathrm{p}_{\mathrm{n}}^{o}=2.1 \times 10^{5} \mathrm{~cm}^{-3} \\
& \mathrm{~A}=1.4 \times 10^{-1} \mathrm{~cm}^{-2}
\end{aligned}
$$

The following observations are made:

1) Behavior of $T_{s / \tau}$ with $I_{\gamma} / I_{f}$ for $\alpha=1$ and $\beta=1$ has been plotted.

2) Behavior of reverse transient waveform for $V(t)$ and $i(t)$ for different values of $R$ have been plotted in Fig 6(a). They predict the value of $I_{s}$ and $t_{f}$ which are close to calculated values.

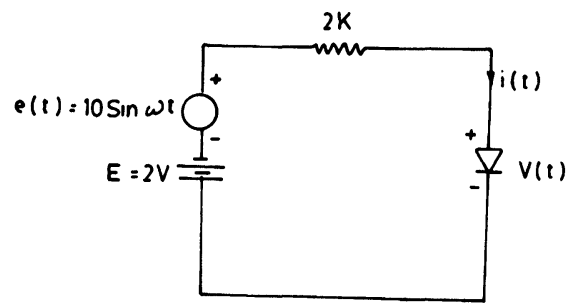

(a)

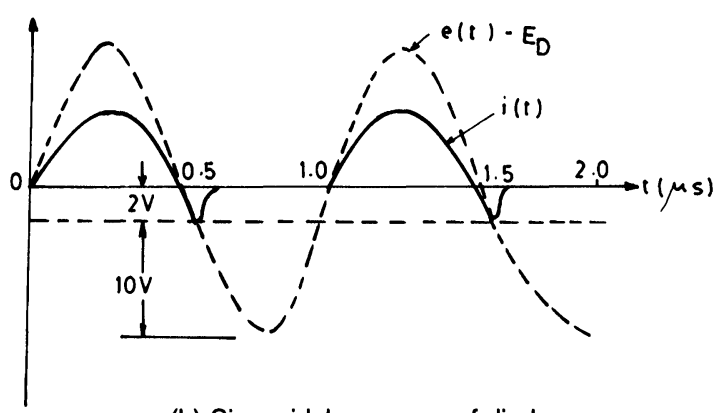

(b) Sinusoidal response of diode

FIGURE 14 
3) Forward transient response of the diode $V(t)$ versus $t$ curve have been plotted in Fig.6 (b) for different values of $I_{f}$. The phenomena of conductivity modulation is observed at high values of $I_{f}$ in form of a voltage bump. This is where the memristor action comes into picture.

\section{Sinusoidal Response in Rectifying Circuits}

The circuits shown in Figs (14 a) and (b) have been simulated in the computer. The results are shown in Figs. (14a) and (b), respectively. We observe that in each case there is a small 'tail' in the current waveform in the cut off cycle. This is obviously due to the effect of the charge stored in the base. 

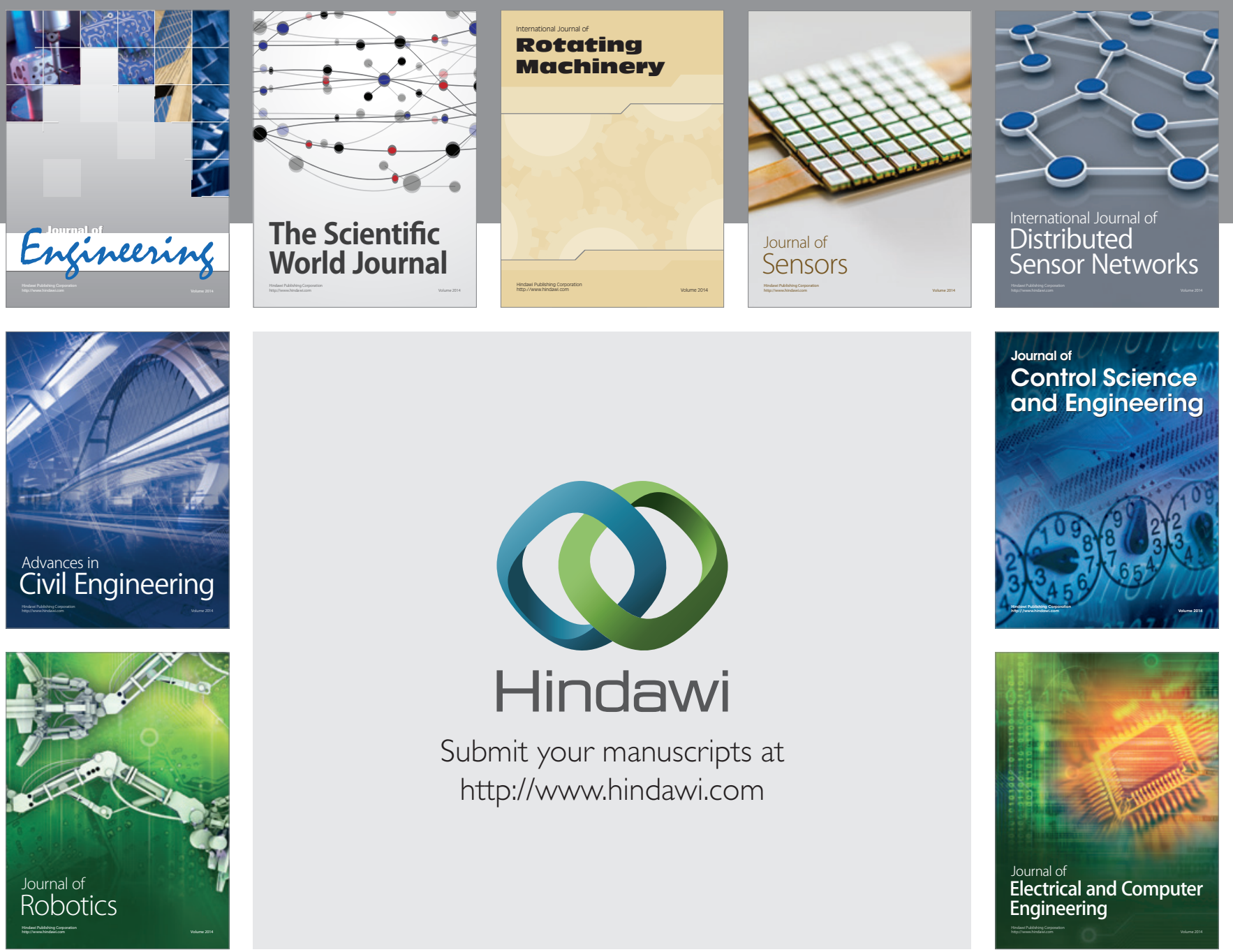

Submit your manuscripts at

http://www.hindawi.com
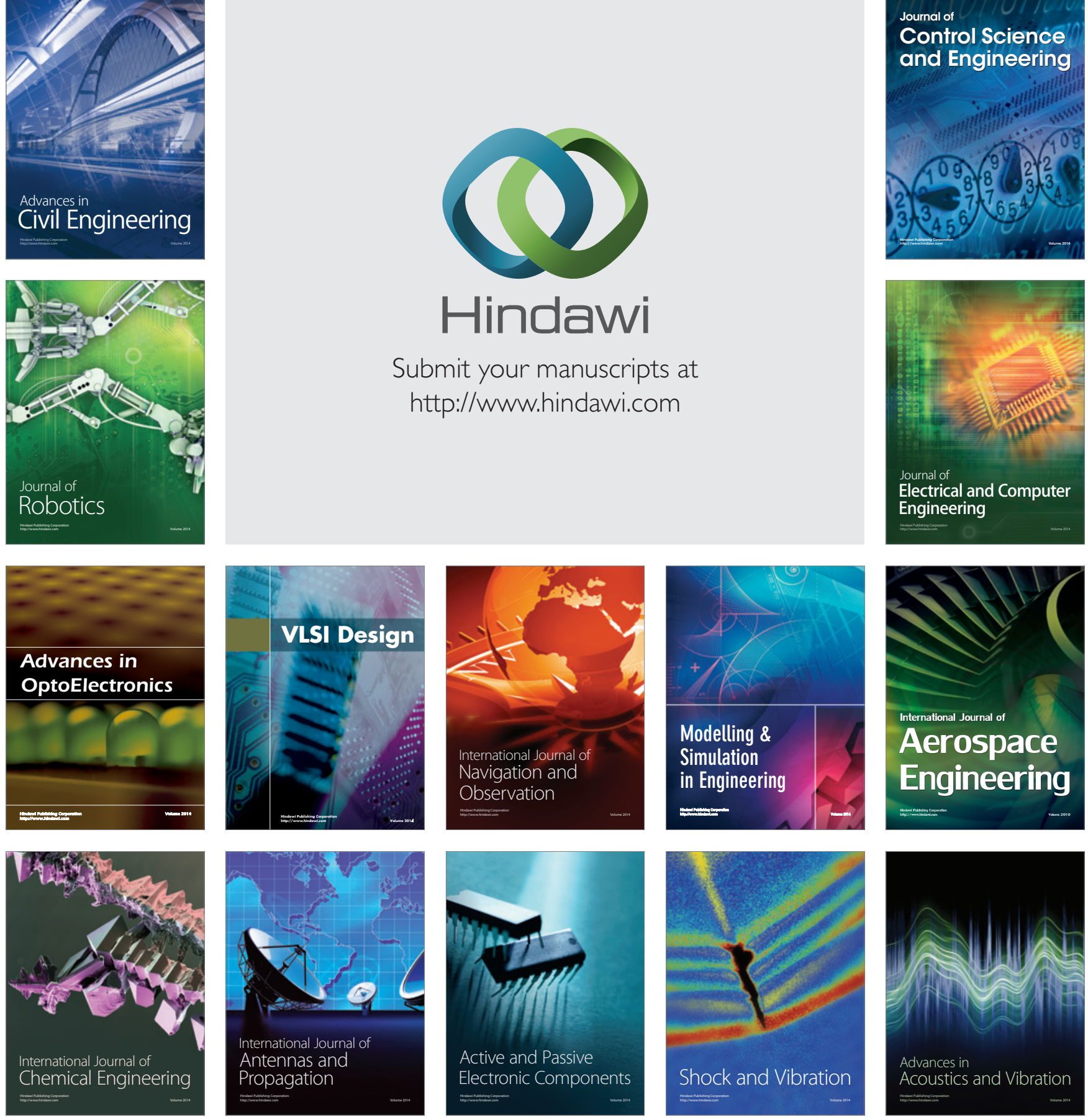